CERN-PH-TH/2009-056

arXiv:0904.4905 [hep-th]

\title{
Calculations for Mirror Symmetry with D-branes
}

\author{
Johannes Walcher \\ PH-TH Division, CERN \\ Geneva, Switzerland
}

\begin{abstract}
We study normal functions capturing D-brane superpotentials on several one- and twoparameter Calabi-Yau hypersurfaces and complete intersections in weighted projective space. We calculate in the B-model and interpret the results using mirror symmetry in the large volume regime, albeit without identifying the precise A-model geometry in all cases. We identify new classes of extensions of Picard-Fuchs equations, as well as a novel type of topology changing phase transition involving quantum D-branes. A 4-d domain wall which is obtained in one region of closed string moduli space from wrapping a four-chain interpolating between two Lagrangian submanifolds is, for other values of the parameters, represented by a disk ending on a single Lagrangian.
\end{abstract}

April 2009 


\section{Contents}

1 Introduction

2 Overview and a Simple Example

2.1 Picard-Fuchs equations . . . . . . . . . . . . . . . . 6

2.2 Extensions . . . . . . . . . . . . . . . . . . . . . . 8

2.3 Example. $\mathbb{P}^{5}[3,3] \ldots \ldots \ldots \ldots$

3 Some More One-parameter Models $\quad 15$

$3.1 \mathbb{P}_{112112}[4,4] \ldots \ldots \ldots \ldots \ldots \ldots \ldots \ldots$

$3.2 \mathbb{P}_{123123}[6,6] \ldots \ldots \ldots \ldots \ldots \ldots$

4 A Two-parameter Model $\quad 20$

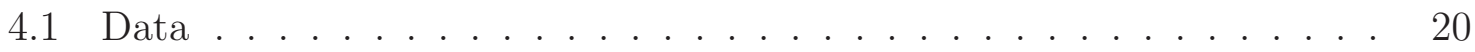

4.2 Curves and residues . . . . . . . . . . . . . . . . . 22

4.3 Solutions . . . . . . . . . . . . . . . . . . . 23

4.4 Mirror map and instanton sum . . . . . . . . . . . . . . . 26

5 Open-Closed Moduli Space of Two-parameter Model 28

6 Restriction to One-parameter Model

7 D-brane Phase Transitions at Large Volume $\quad 33$

7.1 Analytic continuation via dilogarithm . . . . . . . . . . . . 34

7.2 Interpretation . . . . . . . . . . . . . . . . . 35

8 Outlook

Acknowledgments

References 


\section{Introduction}

In this paper, we study the contribution of background D-branes to the spacetime superpotential for closed strings in type II string compactifications on compact CalabiYau threefolds. Our focus is the extension of the methods developed in [1, 2] towards making contact with the standard set of multi-parameter models studied in the context of closed string mirror symmetry, and first in [3, 4, 5, 6, 6 .

The superpotential on the D-brane worldvolume is an interesting quantity to study, from many points of view, and has applications in all areas of D-brane physics and mathematics. Generally speaking, one expects a holomorphic functional $\mathcal{W}(u ; z)$ on the infinitesimal open string state space, coordinatized by $u$, with parametric dependence on closed string moduli $z$. The expansion around the critical points of $\mathcal{W}$ with respect to $u$ should govern the low-energy interactions in the corresponding $\mathcal{N}=1$ string vacuum. This description comes with the important caveat that it is hard to know how to compute invariantly off-shell (or relatedly that the physical couplings depend on the Kähler potential), but is an essential tool towards understanding D-branes on Calabi-Yau manifolds. For a very short sampling of early literature on this subject, see [7, 8, 9, 10, 11].

Dualities shed light on some of these questions. In particular, the relation of open topological strings to Chern-Simons theory and M-theory gives an interpretation of perturbative string amplitudes (of which the superpotential is the tree-level data) in terms of knot invariants and counting of BPS states, respectively [12]. This strategy opened the way to a quantitative understanding of D-brane superpotentials on noncompact geometries [13], subsequently leading to many spectacular developments in topological string theory, see for example [14]. It has also been understood how these superpotential computations fit into a special geometry formalism [15, 16]. On the other hand, these results were, at least initially, restricted to non-compact setups and it has not been clear throughout how the compact case would be covered. More evidence is desirable to further stabilize the status of the superpotential as a numerical invariant of the D-brane configuration space. This should also help to reconnect with the algebraic and categorical approaches developed for instance in [17, 18].

The main lesson of [1, 2] is that already by just restricting the superpotential to the critical points,

$$
\left.\mathcal{W}\right|_{\partial_{u} \mathcal{W}=0}
$$


one obtains a rather non-trivial invariant attached to a general, including compact, D-brane configuration. The quantity (1.1), which depends on discrete open string, and continuous closed string moduli, has a classical mathematical meaning in the B-model [2], as well as an enumerative interpretation in the A-model [19]. Following the quintic, a handful of examples have now been worked out [20, 21]. Progress on the relation to the framework of [16] has also been made, see [22, 23, 24, 25].

In the present paper, we will continue to work with the quantity (1.1), and give examples of some further properties that appear over more complicated (multidimensional) moduli spaces. We will also touch on issues of compactification of moduli, monodromy, and the open extension of the mirror map.

The bulk of our study proceeds by the analysis of examples. We will however begin in section 2 with recalling the basic setup for the computation of the Picard-Fuchs equations in complete intersection Calabi-Yau as well as their extension to the open string sector. As in [2], the D-brane configurations that we study are captured by a collection of holomorphic curves that reside at the intersection of the Calabi-Yau with certain hyperplanes. This discussion will be followed by our first new example, based on the intersection of two cubics in $\mathbb{P}^{5}$. It has the feature that the curves themselves are not complete intersection, but is otherwise qualitatively very similar to [1, 2, 20]. Moreover, the enumerative predictions have been checked in the A-model, giving further support to the entire framework.

We will stay with one-parameter complete intersections in section 3, It has been noted that among the fourteen hypergeometric one-parameter Calabi-Yau Picard-Fuchs equations, ten admit an extension by the same algebraic inhomogeneity as in [1] that is sensible in the sense that the extending solution has an integral Ooguri-Vafa expansion. Of those ten, four are geometrically realized by hypersurfaces in weighted projective space, and the relevant D-brane geometries were identified in A- and B-model in [1, 2, 20, 21]. (The integrality can then be a formal consequence of [26].) We will here supply the B-model branes for most of the remaining cases. Moreover, we will find other algebraic extensions that characterize a different vacuum structure $\left(\mathbb{Z}_{p}\right.$ discrete Wilson line with $p>2$ as opposed to $p=2$ as in [1]). Accompanying the discussion of the two-parameter model, we will also find an extension of the Picard-Fuchs equation in one of the models for which the extension of [1] did not make sense, see section [6. The solution of this last extension is not hypergeometric.

The most involved computations are undertaken for the two-parameter model 
known as $\mathbb{P}_{11226}[12]$, for which analysis we will draw on [3]. We summarize our Dbrane geometry in section 4, and show that we obtain an integral instanton expansion around the appropriate large volume point. In section 5, we discuss the structure of the moduli space. When studying D-branes using (1.1), one expects that the combined open-closed moduli space is generically a multi-covering of the closed string moduli space branched over the discriminant locus over which the (discrete) D-brane moduli space becomes singular. Perhaps the most interesting feature is that this D-brane discriminant, being of codimension one, generically intersects the compactification divisor of the underlying closed string moduli space. This intersection need not be transverse and one expects that interesting physics will take place at these new types of singularities. (Most of the mathematics should be in place, although the singularities of normal functions over multi-parameter moduli spaces remain an active field of research, see, e.g., [27] for a recent survey.)

We will analyze in detail only one of these new structures, where the D-brane discriminant enters the large volume region. The first step here is an additional blowup of the moduli space described in [3]. The coordinate on the exceptional divisor corresponds to the quantum volume (BPS tension) of a domainwall that interpolates between certain vacua of our D-brane geometry. As we will see, there are then two regimes in which the open-closed string background admits a classical geometric interpretation in the A-model. In one of them, the domainwall is represented in the A-model by a 4-chain interpolating between two Lagrangian submanifolds, and in the other, by a disk ending on a single Lagrangian. (In the B-model, the domainwalls are always represented by 3 -chains suspended between holomorphic curves.) The smooth interpolation between the two regimes constitutes a new instance of a topology changing transition, of the type first observed in [28, 29]. Note that we are able to make these assertions without having identified the actual D-brane configuration in the Amodel. We will however discuss which qualitative features this geometry must have in order to be consistent with the B-model and mirror symmetry. A linear sigma model description of the phenomenon would be desirable.

\section{Overview and a Simple Example}

In this paper, we study Calabi-Yau geometries that are obtained from the intersection of the zero locus of a collection of polynomials $\left(W_{j}\right)_{1 \leq j \leq n-3}$ in variables $\left(x_{i}\right)_{1 \leq i \leq n+1}$. 
The $x_{i}$ are homogeneous coordinates on weighted projective space $\mathbb{P}_{w_{1}, \ldots, w_{n+1}}^{n}$, and the $W_{j}$ are assumed to be homogeneous of degree $d_{j}$ with respect to the scaling specified by the $w_{i}$. This means that

$$
v\left(W_{j}\right)=d_{j} W_{j}
$$

where $v$ is the Euler vector field

$$
v=\sum_{i} w_{i} x_{i} \frac{\partial}{\partial x_{i}}
$$

The complete intersection of the $\left\{W_{j}=0\right\}$ is Calabi-Yau if $\sum_{i=1}^{n+1} w_{i}=\sum_{j=1}^{n-3} d_{j}$.

As usual, the A-model geometry, which we will denote by $X$, is obtained by choosing the $W_{j}$ generic transversal, and appropriately resolving the loci where their intersection meets the singularities of the weighted projective space. The A-model then depends on $h_{11}(X) \geq 1$ independent Kähler classes and, at closed string tree level, captures the classical intersection ring $H^{\text {even }}(X)$ together with its quantum corrections due to worldsheet instantons. The B-model geometry, consequently denoted by $Y$, has several equivalent descriptions. We will use the version going back to Greene-Plesser [30] in which $Y$ is the resolution of the quotient of a particular family (of dimension $h_{12}(Y)$ ) of manifolds $\cap\left\{W_{j}=0\right\}$ by a certain maximal discrete group of phase symmetries preserving the Calabi-Yau condition. The observables in the B-model originate mathematically from the variation of Hodge structure associated with the family. Mirror symmetry identifies A- and B-model and, in particular, $h_{11}(X)=h_{12}(Y)$.

We will study in practice only cases with $h_{11}(X)=h_{12}(Y)=1$ or 2 . Just as the results of [2, 20], the structure visible in the examples of the present paper is compatible with an application of methods of toric geometry [31] to compact Calabi-Yau geometries with D-branes. This was recently studied in [24].

\subsection{Picard-Fuchs equations}

The moduli spaces governing closed string mirror symmetry can be studied by computing the periods of the holomorphic three-form on $Y$, i.e.

$$
\varpi(z)=\int_{\Gamma} \Omega
$$

where $\Omega \in H^{3,0}(Y)$, and $\Gamma \in H_{3}(Y ; \mathbb{Z})$. We have here summarily denoted the complex structure moduli of $Y$ by $z$. They appear in certain combinations as parameters in the defining polynomials $W_{j}$. A good deal of information about the periods (2.3) can be 
obtained from the differential equations that they satisfy as functions of the $z$. These differential equations originate from the fact that taking derivatives of $\Omega$ with respect to the parameters generates other elements of the third cohomology $H^{3}(Y)$. The latter being finite-dimensional results in cohomological relations amongst the derivatives of $\varpi(z)$, known as the Picard-Fuchs differential ideal. For such considerations to make sense, it is important that the three-cycles $\Gamma$ against which we integrate the threeforms be topological, i.e., they can be chosen independent of the complex structure parameters $z$.

The periods then satisfy the Picard-Fuchs differential equations, but so does any (z-independent) complex linear combination. Singling out an integral basis requires additional information that can be obtained in part from considerations of monodromy, in particular at points of maximal unipotent monodromy, as well as by comparison with explicit integration around carefully chosen cycles.

A useful algorithm to derive these Picard-Fuchs equations is the Griffiths-Dwork reduction method [32]. For the complete intersections in weighted projective space as described above, we may represent the holomorphic three-form as a residue

$$
\Omega=\frac{|G|}{(2 \pi i)^{3}} \operatorname{Res}_{W_{j}=0} \frac{\omega}{\prod_{j} W_{j}}
$$

where $\omega$ is the $n$-form

$$
\omega=\alpha(v)=\sum_{i}(-1)^{i-1} w_{i} x_{i} d x_{1} \wedge \cdots \widehat{d x}_{i} \cdots \wedge d x_{n+1}
$$

obtained by contracting the "virtual" $n+1$-form

$$
\alpha=d x_{1} \wedge \cdots \wedge d x_{n+1}
$$

with the Euler vector field $v$ from eq. (2.2). In (2.4), we have inserted the order of the discrete group, $G$, that relates the $\left\{W_{j}=0\right\}$ to $Y$, as in e.g., [33]. To make the meaning of the residue in (2.4) more explicit, given a three-cycle $\Gamma \subset \cap_{j}\left\{W_{j}=0\right\}$, we construct a "tube-over-cycle", $T(\Gamma)$, by fibering an $n$-3-dimensional torus over $\Gamma$ that surrounds all $\left\{W_{j}=0\right\}$ sufficiently closely. Then,

$$
\int_{\Gamma} \Omega=\frac{|G|}{(2 \pi i)^{n}} \int_{T(\Gamma)} \frac{\omega}{\prod_{j} W_{i}}
$$

Periods of derivatives of $\Omega$ with respect to the parameters take very similar forms. The fundamental relation that allows the Griffiths-Dwork reduction is the identity between 
meromorphic forms in the ambient (weighted) projective space,

$$
d\left(\frac{A^{i} \omega_{i}}{P}\right)=\frac{\partial_{i} A^{i} \omega}{P}-\frac{A^{i} \partial_{i} P \omega}{P^{2}}
$$

where $P$ is any homogeneous polynomial of degree say $D$, the $A^{i}$ are polynomials of degree $D-\sum_{j \neq i} w_{j}$, and $\omega_{i}$ is the contraction

$$
\omega_{i}=\omega\left(\partial_{i}\right)=\alpha\left(v, \partial_{i}\right)
$$

By analyzing the relations in the polynomial ideal $\mathbb{C}\left[x_{i}\right] / \partial W_{j}$, helped by exploiting the discrete group action, the Griffiths-Dwork method delivers differential operators $\mathcal{L}_{G D}$ together with meromorphic $n-1$-forms $\tilde{\beta}$ such that

$$
\mathcal{L}_{G D}\left(\frac{\omega}{\prod_{j} W_{j}}\right)=d \tilde{\beta}
$$

It is important for us to keep in mind that the explicit form of the $\tilde{\beta}$ depends on a choice of representatives for cohomology and relations.

Of course, for complete intersections of the type described above, the Picard-Fuchs equations can be obtained more efficiently by an appropriate extension of the GKZ differential system associated with the ambient toric variety [34, 5, 6]. The equations are much simpler to solve in the resulting form that exposes the hypergeometric structure of the solutions, so it is convenient to rewrite the equations in this fashion. In the examples, we will chose bases of relations such that the Griffiths-Dwork, $\mathcal{L}_{\mathrm{GD}}$, and hypergeometric, $\mathcal{L}$, operators are simply related by (possibly $z$-dependent) normalization factors $\mathcal{N}_{\mathrm{GD}}$ and $\mathcal{N}$.

$$
\mathcal{L} \mathcal{N}=\mathcal{N}_{\mathrm{GD}} \mathcal{L}_{\mathrm{GD}}
$$

The normalization $\mathcal{N}_{\mathrm{GD}}$ is of course irrelevant for computations of ordinary periods, but becomes essential in the context of open string computations, to which we now turn.

\subsection{Extensions}

The basic idea behind our computations is to study the open string observable (1.1) via its representation as a chain integral

$$
\mathcal{W}_{C}=\int^{C} \Omega
$$


Here, $C \subset Y$ is a holomorphic curve representing the corresponding critical point of $\mathcal{W}$, and it is understood that to really carry out the integral, we should choose a pair of homologically equivalent curves, and integrate $\Omega$ over a bounding three-chain. This corresponds in (1.1) to the computation of BPS domainwall tensions as differences of superpotentials between critical points. Mathematically, such objects are known as normal functions [32]. We refer to [2] for a discussion of the applicability of (2.12).

The chain integral (2.12) is studied via its own differential equations, which is an inhomogeneous extension of the ordinary Picard-Fuchs system. As we will review, the inhomogeneous term results from a local computation around the curve $C$, and does not depend on where we begin the integral. If one is interested in studying domainwalls, and in particular for global consistency over the moduli space, one also needs to fix the solution of the homogeneous equation, that can be freely added to $\mathcal{W}_{C}$, up to integral periods. As for the periods, this requires additional information such as appropriate boundary conditions.

In the paper [2], an interesting D-brane configuration was obtained via some detours as a certain matrix factorization of the (single) polynomial defining a quintic hypersurface. The holomorphic curves needed for the computation of (2.12) were representatives of the second algebraic Chern classes of these matrix factorizations. A similar strategy was pursued in [20, 21] for the other one-parameter hypersurfaces. The selection principle for the matrix factorizations in [2, 20] was a conjectural mirror relation to the Lagrangian submanifolds given as real slices of the corresponding Amodel geometry. (In [21], a different scheme was used, see [35] for a possible extension to multi-parameter models.)

In our examples, we will instead directly specify the curves. We do this first of all because the matrix factorization description of B-branes for complete intersections is more complicated [36], and second of all, because we do not have a confident conjecture about relevant mirror pairs of D-brane configurations. This is due in part to the absence of a Gepner point in the moduli space of these examples where mirror symmetry could have been based on an exactly solvable conformal field theory.

What we will borrow from [2] is that the computation of the inhomogeneous term is possible when the curves are components of the intersection of the $\left\{W_{j}=0\right\}$ with two hyperplanes. (By hyperplane in a weighted projective space, we mean a subspace linear in at least one coordinate.) Thus, in each of our examples, we will choose two 
such hyperplanes, $P_{1}$ and $P_{2}$, such that the intersection

$$
\left(\cap_{j}\left\{W_{j}=0\right\}\right) \cap P_{1} \cap P_{2}=\cup_{i} C_{i}
$$

decomposes into several component curves $C_{i}$. (If there were only one component, the relevant integrals would all vanish automatically.) We will make the construction such that the hyperplanes and the curves deform smoothly as we vary the complex structure parameters z. (More precisely, we allow the possibility of degeneration at co-dimension one discriminant loci, see below.)

The hyperplanes $P_{1}$ and $P_{2}$ are typically not invariant under the action of the discrete group $G$, and the curves on $Y$ really come from the orbits under that action. However, the stabilizer can be non-trivial, and the curves will then intersect the singularities that are resolved in the construction of $Y$. In [2], this was analyzed carefully on the mirror quintic, and it was shown that the net effect is to divide the final result by the order of the stabilizer, $S \subset G$. This amounts to replacing $|G|$ in (2.4) by the length of the orbit, $|O|=|G| /|S|$, to which the respective curve belongs. This is the prescription that we shall assume.

In such a setup, there are in principle two ways to obtain a non-trivial normal function, as observed in [20]. We can compare via the chain integral (2.12) either two component curves in the intersection (2.13), averaged over $G$, or the same components in two different $G$-orbits. In either case, the problem at hand is the computation of the inhomogeneous term that results from the application of a Picard-Fuchs operator to the chain integral (2.12).

So let us finally explain how we compute this inhomogeneous term. We exploit the fact that while $\tilde{\beta}$ in (2.10) is a meromorphic $n-1$-form, the curves in (2.13) are contained in $n-2$-dimensional linear subspaces of the ambient projective space. Thus, by laying the tube around the curves inside of $P_{1} \cap P_{2}$ as much as possible, the computation of

$$
\int_{T\left(C_{i}\right)} \tilde{\beta}
$$

localizes to the points $\left\{p_{1}, p_{2}, \ldots\right\}$ where $C_{i}$ intersects one of the other components. In a local neighborhood $U_{k}$ of each $p_{k}$, we chose a local parameterization of $C_{i}$, and $n-3$ normal vectors $n_{j}$ that point inside of $P_{1} \cap P_{2}$ outside of $U_{k}$. To guarantee that we are surrounding all $\left\{W_{j}=0\right\}$, it is most convenient to arrange the $n_{j}$ such that on $U_{k}$,

$$
\begin{aligned}
& n_{j}\left(W_{j}\right)>0, \\
& n_{i}\left(W_{j}\right)=0, \quad \text { for } i \neq j
\end{aligned}
$$


By perturbing the curve in the direction $\sum \epsilon_{j} n_{j}$, with each $\epsilon_{j}$ encircling the origin in the complex plane, the integral of $\tilde{\beta}$ around $C_{i}$ is computed from a combination of $n-2$ angular integrals and one radial integral, in a simple generalization of [2]. Note that in this residue computation, the angular integrals combine with the $(2 \pi i)^{-n}$ in (2.7) to leave us with an overall $1 /(2 \pi i)^{2}$ characteristic of a normal function associated with a curve on a Calabi-Yau threefold.

We have thus computed

$$
\frac{|O|}{(2 \pi i)^{n}} \int_{T\left(C_{i}\right)} \tilde{\beta}
$$

as a first contribution to the inhomogeneous term in the Picard-Fuchs equation. However, as anticipated, there is in general a second contribution, which originates from the action of the differential operator on the three-chain. (In distinction to three-cycles, the three-chains are not annihilated by the Gauss-Manin connection. Note that there is no invariant separation between the two types of contributions.) As before, this contribution localizes to the curves, and further to the intersection points $p_{k}$. To show how this is done in practice, we let $n_{z}$ be a normal vector representing the first order variation of $C_{i}$ with respect to the complex structure parameter $z$. We then have, for example,

$$
\frac{\partial^{k}}{\partial z^{k}}\left(\int^{C_{i}} \Omega\right)=\sum_{l=1}^{k} \frac{\partial^{k-l}}{\partial z^{k-l}}\left(\int_{C_{i}}\left(\partial_{z}^{l-1} \Omega\right)\left(n_{z}\right)\right)+\int^{C_{i}} \partial_{z}^{k} \Omega
$$

The final term enters the Griffiths-Dwork reduction process, while the intermediate integrals can be computed as residues as described above, and then safely differentiated.

The total inhomogeneity is obtained by collecting (2.16) and terms of the form (2.17). As stressed above, to actually solve the inhomogeneous Picard-Fuchs equation, it is convenient to revert to its hypergeometric form via (2.11).

\subsection{Example. $\mathbb{P}^{5}[3,3]$}

We begin our collection of examples with a model that is qualitatively similar to the quintic studied in [1, 19, 2]. In particular, if we conjecture that the D-brane configurations that we will specify below are mirror to the real slices of the A-model, we obtain enumerative predictions that can be (and in fact have been) checked by independent localization computations in the A-model. The underlying manifold in the A-model is the intersection of two cubics in $\mathbb{C P}^{5}$, which, in the notation of the previous subsections, corresponds to $n=5, w_{1}=\ldots=w_{6}=1$, and $d_{1}=d_{2}=3$. As first observed in 
[37, the mirror manifold can be represented by the quotient of a one-parameter family of bicubics,

$$
Y=\left\{W_{1}=0, W_{2}=0\right\} / G
$$

where $W_{1}$ and $W_{2}$ are the particular cubic polynomials

$$
\begin{aligned}
& W_{1}=\frac{x_{1}^{3}}{3}+\frac{x_{2}^{3}}{3}+\frac{x_{3}^{3}}{3}-\psi x_{4} x_{5} x_{6} \\
& W_{2}=\frac{x_{4}^{3}}{3}+\frac{x_{5}^{3}}{3}+\frac{x_{6}^{3}}{3}-\psi x_{1} x_{2} x_{3}
\end{aligned}
$$

with the complex structure parameter $\psi$, and $G \cong \mathbb{Z}_{3}^{2} \times \mathbb{Z}_{9}$ is the maximal discrete group preserving $W_{1}$ and $W_{2}$, as well as the holomorphic three-form (2.4). By applying Griffiths-Dwork reduction as reviewed above, we obtain the Picard-Fuchs operator [37]

$$
\mathcal{L}_{\mathrm{GD}}=\frac{\psi^{6}-1}{64} \partial_{\psi}^{4}+\frac{7 \psi^{6}-1}{32 \psi} \partial_{\psi}^{3}+\frac{55 \psi^{6}+1}{64 \psi^{2}} \partial_{\psi}^{2}+\frac{65 \psi^{6}-1}{64 \psi^{3}} \partial_{\psi}+\frac{\psi^{2}}{4}
$$

with an inhomogeneous term that in our scheme begins as

$$
\begin{aligned}
\tilde{\beta}= & -\frac{\psi^{2} x_{3} \omega_{3}}{4 W_{1} W_{2}}-\frac{x_{1}^{2} x_{2} x_{3} \omega_{1}}{192 \psi^{3} W_{1}^{2} W_{2}}+\frac{17 \psi^{3} x_{1}^{2} x_{2} x_{3} \omega_{1}}{192 W_{1}^{2} W_{2}}-\frac{x_{1} x_{2}^{2} x_{3} \omega_{2}}{192 \psi^{3} W_{1}^{2} W_{2}} \\
& +\frac{17 \psi^{3} x_{1} x_{2}^{2} x_{3} \omega_{2}}{192 W_{1}^{2} W_{2}}-\frac{\psi^{3} x_{1} x_{2} x_{3}^{2} \omega_{3}}{4 W_{1} W_{2}^{2}}-\frac{x_{1} x_{2} x_{3}^{2} \omega_{3}}{192 \psi^{3} W_{1}^{2} W_{2}}+\frac{17 x_{1} x_{2} x_{3}^{2} \omega_{3}}{192 W_{1}^{2} W_{2}}+\cdots
\end{aligned}
$$

and contains a total of 64 similar such terms. By changing the normalization via (2.11) with $\mathcal{N}=\psi^{2}, \mathcal{N}_{\mathrm{GD}}=4 / 81$, we obtain the hypergeometric differential operator

$$
\mathcal{L}=\theta^{4}-9 z(3 \theta+1)^{2}(3 \theta+2)^{2}
$$

where $z=(3 \psi)^{-6}$, and $\theta=z \frac{d}{d z}$.

Turning to the specification of D-brane configurations, we consider the hyperplanes

$$
P_{1}=\left\{x_{1}+x_{2}=0\right\}, \quad P_{2}=\left\{x_{4}+x_{5}=0\right\}
$$

The intersection of $P_{1}, P_{2}$ with $\left\{W_{1}=0, W_{2}=0\right\}$ is reducible. It contains the line

$$
C_{0}=\left\{x_{1}+x_{2}=0, x_{4}+x_{5}=0, x_{3}=0, x_{6}=0\right\}
$$

as well as two degree 4 curves, $C_{+}$and $C_{-}$. Their homogeneous ideal is generated by

$$
\left\langle x_{1}+x_{2}, x_{4}+x_{5}, x_{3}^{3}+3 \psi x_{4}^{2} x_{6}, x_{6}^{3}+3 \psi x_{1}^{2} x_{3}, x_{3}^{4} \pm 9 \psi^{2} x_{4}^{3} x_{1}, x_{6}^{4} \pm 9 \psi^{2} x_{1}^{3} x_{4}\right\rangle
$$


Notice that these are not complete intersection curves. [The simplest way to understand the curves is via their rational parameterization

$$
\begin{array}{lll}
x_{1}=u^{4}, & x_{2}=-u^{4}, & x_{3}=\alpha_{1} \sqrt{3 \psi} u v^{3} \\
x_{4}=v^{4}, & x_{5}=-v^{4}, & x_{6}=\alpha_{2} \sqrt{3 \psi} u^{3} v
\end{array}
$$

where $(u, v)$ are homogeneous coordinates on $\mathbb{P}^{1}$, and $\alpha_{1}, \alpha_{2}$ are fourth roots of $\mp 1$ satisfying $\alpha_{1}^{3}+\alpha_{2}=\alpha_{2}^{3}+\alpha_{1}=0$.] Implementing the residue computation sketched above (see [2, 20] for more details), we find

$$
\int_{T\left(C_{ \pm}\right)} \tilde{\beta}= \pm(2 \pi i)^{3} \frac{3}{32 \psi^{3}}
$$

The action of the derivatives on the curves (2.17) does not contribute for our choice of tube and $\tilde{\beta}$. Finally, we need to collect the various normalization factors. It is not hard to see that the discrete group acts by relating $P_{1}, P_{2}$ to 9 similar pairs of hyperplanes. The stabilizer of $C_{ \pm}$is $\mathbb{Z}_{9}$. This leads to the inhomogeneous Picard-Fuchs equation in hypergeometric form

$$
\mathcal{L} \int^{C_{ \pm}} \Omega=\frac{ \pm 1}{(2 \pi i)^{2}} \frac{9}{8} \sqrt{z}
$$

We can solve this equation around $z=0$ by recalling the hypergeometric generating function,

$$
\varpi(z ; H)=\sum_{n=0}^{\infty} \frac{\Gamma(1+3(n+H))^{2}}{\Gamma(1+n+H)^{6}} z^{n+H}
$$

Indeed, we have, as a power-series in $z$,

$$
\mathcal{L} \varpi(z ; H)=H^{4} \frac{\Gamma(1+3 H)^{2}}{\Gamma(1+H)^{6}} z^{H}
$$

Since

$$
\mathcal{L} \partial_{H} \varpi(z ; H)=\partial_{H} \mathcal{L} \varpi(z ; H),
$$

we find that the solutions of the homogeneous equation $\mathcal{L} \varpi(z)=0$ are the derivatives with respect to $H$,

$$
\varpi_{k}(z)=\partial_{H}^{k} \varpi(z ; 0) \quad \text { for } k=0,1,2,3
$$

A solution of the inhomogeneous equation (2.28) is given by

$$
\mathcal{W}_{ \pm}(z)= \pm \frac{1}{8} \varpi(z ; 1 / 2)
$$


as can be seen from the identity

$$
\frac{\Gamma(1+3 / 2)^{2}}{\Gamma(1+1 / 2)^{6}}=\frac{36}{\pi^{2}}
$$

We should note that the actual domainwall tension might differ from $\mathcal{W}_{+}-\mathcal{W}_{-}$by a solution of the homogeneous equation. This could be studied by careful analytic continuation and computation of monodromies. The boundary condition at the point $\psi=0$ is somewhat more delicate to understand than on the quintic, since the manifold (2.18) becomes singular there.

An A-model interpretation of the superpotential contribution (2.33) can be obtained as usual by expanding the normalized expression

$$
\hat{\mathcal{W}}_{ \pm}(q)=(2 \pi i)^{2} \frac{\mathcal{W}_{ \pm}(z(q))}{\varpi_{0}(z(q))}
$$

( $\varpi_{0}$ is an integral period in the normalization (2.4), see [33].) in the appropriate flat coordinates

$$
q=e^{2 \pi i t}=\exp \left(\frac{\varpi_{1}}{\varpi_{0}}(z)\right)
$$

The first few terms are

$$
\pm \hat{\mathcal{W}}_{ \pm}=18 q^{1 / 2}+182 q^{3 / 2}+\frac{787968}{25} q^{5 / 2}+\cdots
$$

By the methods of [19], it is known that the entire series $\hat{\mathcal{W}}$ reproduces the open Gromov-Witten invariants counting maps from the disk to $(X, L)$, where $L$ is the real slice of the intersection $X$ of two generic cubics in $\mathbb{P}^{5}$. Notice that for $X=\left\{W_{1}=\right.$ $\left.0, W_{2}=0\right\}$ at $\psi=0, L \cong \mathbb{R P}^{3}$, just as on the quintic.

Finally, we expand $\hat{\mathcal{W}}$ using the multi-cover formula,

$$
\hat{\mathcal{W}}_{ \pm}= \pm \sum_{d, k \text { odd }} \frac{N_{d}}{k^{2}} q^{k d / 2}
$$

obtaining $N_{1}=18, N_{3}=180, N_{5}=31518, \ldots$ According to the proposal of [12, 1], the $N_{d}$ count the degeneracy of BPS domainwalls separating the two 4 -d $\mathcal{N}=1$ vacua corresponding to the choice of discrete Wilson line on $L \cong \mathbb{R} \mathbb{P}^{3}$. Integrality of the $N_{d}$ as a mathematical theorem follows, as on the quintic, from the recent results of [26]. Real enumerative invariants in the sense of [38] are given by $N_{d} / 2$. 


\section{Some More One-parameter Models}

Before turning to the two-parameter model, we present here two more one-parameter models with hypergeometric Picard-Fuchs equation. These are the simplest examples for which we have found inhomogeneous terms different from the simple $\sqrt{z}$ extension prominent in all previously studied cases. We will be content with solving the inhomogeneous Picard-Fuchs equations up to rational periods, and not work out the exact spectrum of domainwalls.

Closed string mirror symmetry for both models is discussed in detail in [39]. To abbreviate some of the formulas below, we introduce the hypergeometric generating function

$$
\varpi(z ; H)=\sum_{n=0}^{\infty} \frac{\prod_{j=1}^{n-3} \Gamma\left(1+d_{j}(n+H)\right)}{\prod_{i=1}^{n+1} \Gamma\left(1+w_{i}(n+H)\right)} z^{n+H}
$$

for given weights $w_{i}$ of the homogeneous coordinates, and degrees $d_{j}$ of the defining polynomials. It is also convenient to reserve a notation for the coefficient of the lowest order term of $\mathcal{L} \varpi(z ; H)$

$$
\Xi(H)=H^{4} \frac{\prod_{j=1}^{n-3} \Gamma\left(1+d_{j} H\right)}{\prod_{i=1}^{n+1} \Gamma\left(1+w_{i} H\right)}
$$

\section{$3.1 \quad \mathbb{P}_{112112}[4,4]$}

The B-model geometry is determined by

$$
\begin{aligned}
& W_{1}=\frac{x_{1}^{4}}{4}+\frac{x_{2}^{4}}{4}+\frac{x_{3}^{2}}{2}-\psi x_{4} x_{5} x_{6} \\
& W_{2}=\frac{x_{4}^{4}}{4}+\frac{x_{5}^{4}}{4}+\frac{x_{6}^{2}}{2}-x_{1} x_{2} x_{3}
\end{aligned}
$$

and $G \cong \mathbb{Z}_{2}^{2} \times \mathbb{Z}_{16}$. The generators of $G$ can be taken to be, in customary notation, [39]

$$
\frac{1}{2}(0,1,1,0,0,0), \quad \frac{1}{2}(0,0,0,0,1,1), \quad \frac{1}{16}(0,4,0,1,13,2)
$$

Griffiths-Dwork reduction with $\mathcal{N}=\psi, \mathcal{N}_{\mathrm{GD}}=1 / 64 \psi$ produces the Picard-Fuchs operator

$$
\theta^{4}-16 z(4 \theta+1)^{2}(4 \theta+3)^{2}
$$

where $z=(8 \psi)^{-4}$. 
(i) We first exhibit the curves that produce the $\sqrt{z}$ extension familiar from the quintic. Consider the hyperplanes

$$
P_{1}=\left\{x_{1}+\alpha_{1} x_{2}\right\} \quad P_{2}=\left\{x_{4}+\alpha_{2} x_{5}=0\right\}
$$

where $\alpha_{1}, \alpha_{2}$ are fourth roots of -1 . These 16 pairs of hyperplanes decompose into two orbits of length 8 under the action of $G$. The intersection of $W_{1}=0, W_{2}=0$ with $P_{1}, P_{2}$ splits into two components,

$$
\begin{aligned}
& C_{+}=P_{1} \cap P_{2} \cap\left\{x_{3}=0, x_{6}=0\right\} \\
& C_{-}=P_{1} \cap P_{2} \cap\left\{x_{3}^{3}+8 \psi^{2} \alpha_{2}^{2} \alpha_{1} x_{4}^{4} x_{2}^{2}=0, x_{2}^{2}+2 \psi \alpha_{2} x_{4}^{2} x_{6}=0, x_{6}^{2}+2 \alpha_{1} x_{2}^{2} x_{3}=0\right\}
\end{aligned}
$$

The residue computation delivers

$$
\mathcal{L}_{\mathrm{GD}} \int^{C_{ \pm}} \Omega= \pm \frac{|O|}{(2 \pi i)^{2}} \frac{1}{2 \psi}
$$

and gives the inhomogeneous Picard-Fuchs equation

$$
\mathcal{L} \mathcal{W}_{ \pm}(z)= \pm \frac{4}{(2 \pi i)^{2}} \sqrt{z}
$$

As in the previous cases, this is solved by

$$
\mathcal{W}_{ \pm}= \pm \frac{1}{4} \varpi(z ; 1 / 2)
$$

The normalization again comes out thanks to

$$
\Xi(1 / 2)=\frac{1}{2^{4}} \frac{\Gamma(1+2)^{2}}{\Gamma(1+1 / 2)^{4} \Gamma(1+1)^{2}}=\frac{4}{\pi^{2}}
$$

The low-degree BPS invariants computed as above (2.38) are $N_{1}=64, N_{3}=5568$, $N_{5}=4668864, \ldots$ It is possible that these can be interpreted as real enumerative invariants as for the previously studied models [1, 20, 21]. Note also that since we have two $G$-orbits in the set of planes described above, the collection of curves shares a second domainwall that is a rational linear combination of closed string periods. This is similar to an observation on the degree 8 hypersurface in [20].

(ii) Now consider the hyperplanes

$$
P_{1}=\left\{x_{1}^{2}+\alpha_{1} \sqrt{2} x_{3}\right\}, \quad P_{2}=\left\{x_{4}^{2}+\alpha_{2} \sqrt{2} x_{6}\right\}
$$


where $\alpha_{1}, \alpha_{2}= \pm i$. All pairs are related by the action of $G$, so there is only one orbit, of length 4 . The intersection with $W_{1}=0, W_{2}=0$ splits into 4 components,

$$
\begin{aligned}
& C_{0}=P_{1} \cap P_{2} \cap\left\{x_{2}=0, x_{5}=0\right\} \\
& C_{\eta}=P_{1} \cap P_{2} \cap\left\{W_{1}=0, W_{2}=0, x_{2}^{5}=\eta \frac{2^{5 / 2} \psi^{4 / 3}}{\left(-\alpha_{2}^{4} \alpha_{1}\right)^{1 / 3}} x_{4}^{4} x_{1}\right\}
\end{aligned}
$$

where $\eta$ is a third root of unity keeping track of the root of $\psi$ we are taking. This pattern of curve is signaling a $\mathbb{Z}_{3}$-symmetric collection of brane vacua. Indeed, the residue computation gives

$$
\mathcal{L}_{\mathrm{GD}} \int^{C_{\eta}} \Omega=\frac{|O|}{(2 \pi i)^{2}}\left(\frac{8}{27 \eta^{2} \psi^{1 / 3}}+\frac{50}{27 \eta \psi^{5 / 3}}\right)
$$

[We also find

$$
\mathcal{L}_{\mathrm{GD}} \int^{C_{0}} \Omega=0
$$

as necessary.] Transformation to hypergeometric form yields

$$
\mathcal{L} \int^{C_{\eta}} \Omega=\frac{1}{(2 \pi i)^{2}}\left(\frac{8}{27} \eta z^{1 / 3}+\frac{800}{27} \eta^{2} z^{2 / 3}\right)
$$

Noticing that

$$
\Xi(1 / 3)=\frac{1}{3 \pi^{2}}, \quad \Xi(2 / 3)=\frac{100}{3 \pi^{2}}
$$

(where $\Xi$ is defined in $(\underline{3.2})$ ) we see that the solution can be expressed as

$$
\mathcal{W}_{\eta}=\frac{2}{9}\left(\eta \varpi(z ; 1 / 3)+\eta^{2} \varpi(z ; 2 / 3)\right)
$$

We can now apply the standard mirror map to obtain the A-model expansion

$$
\hat{\mathcal{W}}_{\eta}=(2 \pi i)^{2} \frac{\mathcal{W}(z(q))}{\varpi_{0}(z(q))}=24 \eta q^{1 / 3}+150 \eta^{2} q^{2 / 3}+\frac{2571}{2} \eta^{4} q^{4 / 3}+\frac{417024}{25} \eta^{5} q^{5 / 3}+\cdots
$$

We see that the multi-cover formula in the present case takes the form

$$
\hat{\mathcal{W}}_{\eta}=\sum_{3 \nmid d, k} \frac{N_{d}}{k^{2}} \eta^{k d} q^{k d / 3}
$$

with integral $N_{d}$ (as far as we have checked) counting domainwall degeneracies. Understanding the precise geometric meaning of these invariants depends on identifying the A-model geometry mirror to our curves $C_{\eta}$. The symmetry suggests that the corresponding Lagrangians $L$ have a factor $\mathbb{Z}_{3} \subset H_{1}(L)$ in their first homology group, and $\eta$ is a discrete Wilson line. It seems unlikely (although it cannot be excluded) that these can be described as real slices of the complete intersection (3.3). The first few non-trivial numbers are $N_{1}=24, N_{2}=144, N_{4}=1248, N_{5}=16680, \ldots$. 


\section{$3.2 \quad \mathbb{P}_{123123}[6,6]$}

Here, the B-model geometry is determined by

$$
\begin{aligned}
& W_{1}=\frac{x_{1}^{6}}{6}+\frac{x_{2}^{3}}{3}+\frac{x_{3}^{2}}{2}-\psi x_{4} x_{5} x_{6} \\
& W_{2}=\frac{x_{4}^{6}}{6}+\frac{x_{5}^{3}}{3}+\frac{x_{6}^{2}}{2}-x_{1} x_{2} x_{3}
\end{aligned}
$$

and $G \cong \mathbb{Z}_{36}$, whose generator we take to be

$$
g=\frac{1}{36}(0,24,18,1,14,21)
$$

Griffiths-Dwork reduction with $\mathcal{N}_{\text {hyper }}=\psi, \mathcal{N}_{\mathrm{GD}}=1 / 324 \psi^{3}$ produces the Picard-Fuchs operator

$$
\mathcal{L}=\theta^{4}-144 z(6 \theta+1)^{2}(6 \theta+5)^{2}
$$

with $z=4^{-1}(6 \psi)^{-6}$.

(i) We here begin with the set of hyperplanes

$$
P_{1}=\left\{x_{1}^{2}+2^{1 / 3} \alpha_{1} x_{2}=0\right\}, \quad P_{2}=\left\{x_{4}^{2}+2^{1 / 3} \alpha_{2} x_{5}=0\right\}
$$

where $\alpha_{1}, \alpha_{2}$ are third roots of -1 . The curves are

$$
\begin{aligned}
& C_{0}=P_{1} \cap P_{2} \cap\left\{x_{3}=0, x_{6}=0\right\} \\
& C_{\eta}=P_{1} \cap P_{2} \cap\left\{W_{1}=0, W_{2}=0, x_{3}=\eta \frac{2^{2 / 3} \psi^{2 / 3}}{\left(-\alpha_{2}^{2} \alpha_{1}\right)^{1 / 3}} x_{4}^{2} x_{1}\right\}
\end{aligned}
$$

where $\eta$ runs over third roots of unity. These curves have residue (up to an overall phase that depends on the choice of hyperplane, see discussion in the next paragraph)

$$
\begin{aligned}
\mathcal{L}_{\mathrm{GD}} \int^{C_{0}} \Omega & =\frac{|O|}{(2 \pi i)^{2}}\left(-2^{1 / 3} \psi-\frac{9}{2^{1 / 3} \psi}\right) \\
\mathcal{L}_{\mathrm{GD}} \int^{C_{\eta}} \Omega & =\frac{|O|}{(2 \pi i)^{2}}\left(\frac{2^{1 / 3}}{3} \psi+\frac{3}{2^{1 / 3} \psi}\right)
\end{aligned}
$$

The group $G \cong \mathbb{Z}_{36}$ organizes the planes (3.24) into 3 orbits of length 3 . In this example, $G$ also acts on the $\eta$-label in a non-trivial fashion. If $g$ is a generator as in (3.22), then $g^{3}$ acts within a given hyperplane by $\eta \rightarrow e^{2 \pi i / 3} \eta$. Note that this symmetry is consistent with the residues (3.26) being independent of $\eta$. Another consistency check on (3.26) is that the sum of residues over all curves in a given hyperplane vanishes. In 
the end, the $C_{\eta}$ come in 3 orbits of length 9 , while the $C_{0}$ come in 3 orbits of length 3. This translates into

$$
\mathcal{L} \int^{C_{\eta}} \Omega=-\mathcal{L} \int^{C_{0}} \Omega=\frac{1}{(2 \pi i)^{2}}\left(\frac{2}{3} \tilde{\eta} z^{1 / 3}+216 \tilde{\eta}^{2} z^{2 / 3}\right)
$$

We have here reintroduced third roots of unity $\tilde{\eta}$ that keep track of the orbit of planes. In other words, $\tilde{\eta}$ depends on a combination of $\alpha_{1}, \alpha_{2}$ in (3.24). In the present model,

$$
\Xi(1 / 3)=\frac{3}{4 \pi^{2}}, \quad \Xi(2 / 3)=\frac{243}{\pi^{2}} .
$$

So a solution of the inhomogeneous Picard-Fuchs equation can be written as

$$
\mathcal{W}_{\tilde{\eta}}=\frac{2}{9}\left(\tilde{\eta} \varpi(z ; 1 / 3)+\tilde{\eta}^{2} \varpi(z ; 2 / 3)\right)
$$

The A-model interpretation is the same as around (3.20), with $N_{1}=54, N_{2}=1080$, $N_{4}=216432, N_{5}=10094490, \ldots$

(ii) Finally, we intersect with

$$
P_{1}=\left\{x_{1}^{3}+\alpha_{1} \sqrt{3} x_{3}=0\right\}, \quad P_{2}=\left\{x_{4}^{3}+\alpha_{2} \sqrt{3} x_{6}=0\right\}
$$

where $\alpha_{1}, \alpha_{2}= \pm i$. There are now 5 curves in each hyperplane,

$$
\begin{aligned}
& C_{0}=P_{1} \cap P_{2} \cap\left\{x_{2}=0, x_{5}=0\right\} \\
& C_{\eta}=P_{1} \cap P_{2} \cap\left\{W_{1}=0, W_{2}=0, x_{2}^{2}=\eta \frac{\sqrt{3} \psi^{3 / 4}}{\left(\alpha_{2}^{3} \alpha_{1}\right)^{1 / 4}} x_{4}^{3} x_{1}\right\}
\end{aligned}
$$

where $\eta$ is a fourth root of unity.

$$
\begin{aligned}
& \mathcal{L}_{\mathrm{GD}} \int^{C_{0}} \Omega=\frac{|O|}{(2 \pi i)^{2}} 6 \\
& \mathcal{L}_{\mathrm{GD}} \int^{C_{\eta}} \Omega=\frac{|O|}{(2 \pi i)^{2}}\left(-\frac{9 \sqrt{3} \eta^{2} \psi^{3 / 2}}{32}-\frac{3}{2}-\frac{147 \sqrt{3}}{32 \eta^{2} \psi^{3 / 2}}\right)
\end{aligned}
$$

Here, $G$ organizes the hyperplanes (3.30) into 2 orbits of length 2. $g^{2}$ acts within a given plane by $\eta \rightarrow-\eta$. So the $C_{\eta}$ end up in 4 orbits of length 4 , and $C_{0}$ in 2 orbits of length 2. Thus,

$$
\begin{aligned}
& \mathcal{L} \int^{C_{0}} \Omega=\frac{1}{(2 \pi i)^{2}} 16 \sqrt{z} \\
& \mathcal{L} \int^{C_{\eta}} \Omega=\frac{1}{(2 \pi i)^{2}}\left(\frac{1}{8} \eta^{2} z^{1 / 4}-8 \sqrt{z}+882 \eta^{2} z^{3 / 4}\right)
\end{aligned}
$$


The specialization of the hypergeometric coefficient (3.2) here gives

$$
\Xi(1 / 4)=\frac{1}{8 \pi^{2}}, \quad \Xi(1 / 2)=\frac{16}{\pi^{2}}, \quad \Xi(3 / 4)=\frac{882}{\pi^{2}}
$$

This matches the relative coefficients between $z^{1 / 4}$ and $z^{3 / 4}$ in (3.33), while the coefficient of $z^{1 / 2}$ is matched in a linear combination of the $C_{0}$ and $C_{\eta}$. To work out the entire spectrum of domainwall, it would be natural to use the two linearly independent solutions

$$
\begin{aligned}
& \mathcal{W}_{ \pm}= \pm \frac{1}{4} \varpi(z ; 1 / 2) \\
& \mathcal{W}_{\tilde{\eta}}=\frac{1}{4}\left(\tilde{\eta} \varpi(z ; 1 / 4)+\tilde{\eta}^{2} \varpi(z ; 1 / 2)+\tilde{\eta}^{3} \varpi(z ; 3 / 4)\right)
\end{aligned}
$$

where $\tilde{\eta}$ is a fourth root of unity. The Ooguri-Vafa expansion for the two types is, respectively

$$
\begin{aligned}
& \hat{\mathcal{W}}_{ \pm}= \pm \sum_{2 \nmid d, k} \frac{N_{2, d}}{k^{2}} q^{d k / 2} \\
& \hat{\mathcal{W}}_{\tilde{\eta}}=\sum_{4 \nmid d, k} \frac{N_{4, d}}{k^{2}} \tilde{\eta}^{d k} q^{d k / 4}
\end{aligned}
$$

The first few invariants are

$$
\begin{aligned}
& N_{2,1}=256, N_{2,3}=1742592, N_{2,5}=65066366720, \ldots \\
& N_{4,1}=32, N_{4,2}=248, N_{4,3}=2784, N_{4,5}=83680, N_{4,6}=1741896, \ldots
\end{aligned}
$$

\section{A Two-parameter Model}

We now begin our investigation of D-brane superpotentials in the much-studied twoparameter model $\mathbb{P}_{11226}[12]$. For the geometry of the closed string moduli space, we rely on the treatment in $[3,5]$.

\subsection{Data}

The generic degree 12 hypersurface in $\mathbb{P}_{11226}$ meets the singularities of the weighted projective space in a curve along which we have to blow up to produce the A-model geometry $X$. The resolution of singularities can be understood by giving the charges of the gauged linear sigma model fields

\begin{tabular}{c|cccccc|c} 
& $x_{1}$ & $x_{2}$ & $x_{3}$ & $x_{4}$ & $x_{5}$ & $x_{6}$ & $P$ \\
\hline$h_{1}$ & 0 & 0 & 1 & 1 & 3 & 1 & -6 \\
$h_{2}$ & 1 & 1 & 0 & 0 & 0 & -2 & 0
\end{tabular}


We will slightly depart from the notation of [3], and denote by $H_{1}$ the divisor class of $x_{3}=0, H_{2}$ the class of $x_{1}=0$. The exceptional divisor is $E=H_{1}-2 H_{2}$, and $h_{1}, h_{2}$ are the dual curve classes. We recall the classical intersection relations

$$
\begin{aligned}
& H_{2}^{2}=0, \quad H_{1}^{3}=4, \quad H_{1}^{2} H_{2}=2 \\
& 2 h_{1}=H_{1} H_{2}, \quad 2 h_{2}=H_{1}^{2}-2 H_{1} H_{2}
\end{aligned}
$$

The mirror manifold is the two-parameter family of Calabi-Yau threefolds obtained from the vanishing locus of the defining polynomial

$$
W=\frac{x_{1}^{12}}{12}+\frac{x_{2}^{12}}{12}+\frac{x_{3}^{6}}{6}+\frac{x_{4}^{6}}{6}+\frac{x_{5}^{2}}{2}-\psi x_{1} x_{2} x_{3} x_{4} x_{5}-\frac{\phi}{6} x_{1}^{6} x_{2}^{6}
$$

after orbifolding with respect to the maximal group of phase symmetries $G=\mathbb{Z}_{6} \times$ $\mathbb{Z}_{6} \times \mathbb{Z}_{2}$. One may work with the generators

$$
\frac{1}{6}(0,5,1,0,0), \quad \frac{1}{6}(0,5,0,1,0), \quad \frac{1}{2}(0,1,0,0,1)
$$

The periods of the model are governed by a system of two Picard-Fuchs equations. The Griffiths-Dwork algorithm gives the following relations

$$
\begin{aligned}
& \mathcal{L}_{\mathrm{GD}, 1}=-\frac{1}{6} \partial_{\psi}^{3}+\psi^{5} \partial_{\psi}^{2} \partial_{\phi}+\frac{1}{2 \psi} \partial_{\psi}^{2}+3 \psi^{4} \partial_{\psi} \partial_{\phi}-\frac{1}{2 \psi^{2}} \partial_{\psi}+\psi^{3} \partial_{\phi} \\
& \mathcal{L}_{\mathrm{GD}, 2}=18\left(\phi^{2}-1\right) \partial_{\phi}^{2}+\frac{\psi^{2}}{2} \partial_{\psi}^{2}+6 \psi \phi \partial_{\psi} \partial_{\phi}+\frac{3 \psi}{2} \partial_{\psi}+24 \phi \partial_{\phi}+\frac{1}{2}
\end{aligned}
$$

Together with appropriate three-forms $\tilde{\beta}_{1}, \tilde{\beta}_{2}$ whose explicit form we shall suppress. The transformation to hypergeometric form is accomplished by conjugating the $\mathcal{L}_{\mathrm{GD}, i}$ as in (2.11) with $\mathcal{N}_{1}=\mathcal{N}_{2}=\psi$, and

$$
\mathcal{N}_{\mathrm{GD}, 1}=\frac{\phi}{36 \psi^{2}}, \quad \mathcal{N}_{\mathrm{GD}, 2}=\frac{\psi}{72}
$$

One obtains

$$
\begin{aligned}
& \mathcal{L}_{1}=\theta_{1}^{2}\left(\theta_{1}-2 \theta_{2}\right)-8 z_{1}\left(6 \theta_{1}+1\right)\left(6 \theta_{1}+3\right)\left(6 \theta_{1}+5\right) \\
& \mathcal{L}_{2}=\theta_{2}^{2}-z_{2}\left(2 \theta_{2}-\theta_{1}\right)\left(2 \theta_{2}-\theta_{1}+1\right)
\end{aligned}
$$

with $z_{1}=-3^{-3} 2^{-6} \phi \psi^{-6}, z_{2}=(2 \phi)^{-2}$, and $\theta_{i}=z_{i} \frac{d}{d z_{i}}$. The solutions of (4.7) as power series around $z_{1}=z_{2}=0$ can be obtained from the hypergeometric generating function,

$$
\begin{aligned}
& \varpi\left(z_{1}, z_{2} ; H_{1}, H_{2}\right)=\sum_{n_{1}, n_{2}=0}^{\infty} z_{1}^{n_{1}+H_{1}} z_{2}^{n_{2}+H_{2}} \\
& \frac{\Gamma\left(1+6\left(n_{1}+H_{1}\right)\right)}{\left.\Gamma\left(1+n_{2}+H_{2}\right)^{2} \Gamma\left(1+n_{1}+H_{1}\right)^{2} \Gamma\left(1+3\left(n_{1}+H_{1}\right)\right) \Gamma\left(1+n_{1}+H_{1}-2\left(n_{2}+H_{2}\right)\right)\right)}
\end{aligned}
$$


by differentiation,

$$
\begin{aligned}
\varpi_{0} & =\varpi\left(z_{1}, z_{2} ; 0,0\right) \\
\varpi_{h_{1}} & =\partial_{H_{1}} \varpi\left(z_{1}, z_{2} ; 0,0\right) \\
\varpi_{h_{2}} & =\partial_{H_{2}} \varpi\left(z_{1}, z_{2} ; 0,0\right) \\
\varpi_{H_{1}} & =\left(2 \partial_{H_{1}}^{2}+2 \partial_{H_{1}} \partial_{H_{2}}\right) \varpi\left(z_{1}, z_{2} ; 0,0\right) \\
\varpi_{H_{2}} & =\partial_{H_{1}}^{2} \varpi\left(z_{1}, z_{2} ; 0,0\right) \\
\varpi_{X} & =-\left(\frac{2}{3} \partial_{H_{1}}^{3}+\partial_{H_{1}}^{2} \partial_{H_{2}}\right) \varpi\left(z_{1}, z_{2} ; 0,0\right)
\end{aligned}
$$

Recall how these solutions reflect the GLSM charges (4.1) and the intersection relations (4.2). The closed string mirror map around the large volume point identifies the Kähler parameters as

$$
h_{1}=t_{1}=\frac{1}{2 \pi i} \frac{\varpi_{h_{1}}}{\varpi_{0}}, \quad h_{2}=t_{2}=\frac{1}{2 \pi i} \frac{\varpi_{h_{2}}}{\varpi_{0}}
$$

\subsection{Curves and residues}

As in the one-parameter examples studied in the previous sections, there are several possibilities for intersecting (4.3) with two hyperplanes such that the resulting plane curve splits in a non-trivial way in several components, thus realizing the basic pattern of [2]. Presently the most interesting curves are those obtained from the hyperplanes

$$
P_{1}=\left\{x_{3}+2^{-1 / 6} \alpha_{1} x_{1}^{2}=0\right\}, \quad P_{2}=\left\{x_{4}+2^{-1 / 6} \alpha_{2} x_{2}^{2}=0\right\}
$$

where $\alpha_{1}, \alpha_{2}$ are sixth roots of -1 . The intersection of $P_{1} \cap P_{2}$ with $\{W=0\}$ splits in two components,

$$
\left\{x_{5}=\alpha_{ \pm} x_{1}^{3} x_{2}^{3}\right\}
$$

where $\alpha_{ \pm}$are the two solutions of the quadratic equation

$$
\alpha_{ \pm}^{2}-2^{2 / 3} \psi \alpha_{1} \alpha_{2} \alpha_{ \pm}-\frac{\phi}{3}=0
$$

In a way by now familiar, the discrete group $G$ permutes the planes in (4.11). There are 3 orbits of length 12 . We will label the resulting curves by $C_{(\eta, \pm)}$, where $\eta$ is a third root of unity encoding the orbit of planes, and \pm refers to the choice of root in (4.13).

Computation of the residues for the two relations in (4.5) gives

$$
\begin{aligned}
\mathcal{L}_{\mathrm{GD}, 1} \int^{C_{(\eta, \pm)}} \Omega & =-\frac{|O|}{(2 \pi i)^{2}} \frac{2^{5 / 3} \eta \psi^{3}}{3\left(2 \alpha_{ \pm}-2^{2 / 3} \eta \psi\right)^{3}} \\
\mathcal{L}_{\mathrm{GD}, 2} \int^{C_{(\eta, \pm)}} \Omega & =\frac{|O|}{(2 \pi i)^{2}} \frac{2^{5 / 3} \eta}{2 \alpha_{ \pm}-2^{2 / 3} \eta \psi}
\end{aligned}
$$


Collecting all the factors, and solving (4.13), we can transform to hypergeometric form

$$
\begin{aligned}
& \mathcal{L}_{1} \int^{C_{(\eta, \pm)}} \Omega= \pm \frac{1}{(2 \pi i)^{2}} \frac{4 \eta y}{6(1-4 \eta y)^{3 / 2}} \\
& \mathcal{L}_{2} \int^{C_{(\eta, \pm)}} \Omega= \pm \frac{1}{(2 \pi i)^{2}} \frac{1}{3(1-4 \eta y)^{1 / 2}}
\end{aligned}
$$

where we have introduced the variable

$$
y=\left(\frac{z_{1}}{z_{2}}\right)^{1 / 3}=-\frac{2^{2 / 3} \phi}{12 \psi^{2}}
$$

This combination will play a crucial role in the following discussion. Its precise geometric role will be clarified during our discussion of the combined open-closed moduli space in section 5. For now, we proceed with solving (4.15).

\subsection{Solutions}

To get a good power series expansion, we transform to the independent variables $z_{2}, y$. We work with $\eta=1, \pm=+$, and absorb the factor of $(2 \pi i)^{2}$ into $\mathcal{W} \sim \int^{C} \Omega$. With $\theta_{y}=y \frac{d}{d y}$, we have

$$
\begin{aligned}
& 9 \mathcal{L}_{1} \mathcal{W}=\left(\theta_{y}^{2}\left(\theta_{y}-2 \theta_{2}\right)-72 y^{3} z_{2}\left(2 \theta_{y}+1\right)\left(2 \theta_{y}+3\right)\left(2 \theta_{y}+5\right)\right) \mathcal{W}=3 \frac{4 y}{2(1-4 y)^{3 / 2}} \\
& 9 \mathcal{L}_{2} \mathcal{W}=\left(\left(\theta_{y}-3 \theta_{2}\right)^{2}-9 z_{2}\left(2 \theta_{2}-\theta_{y}\right)\left(2 \theta_{2}-\theta_{y}+1\right)\right) \mathcal{W}=3 \frac{1}{(1-4 y)^{1 / 2}}
\end{aligned}
$$

This form of the equations gives us the opportunity to verify, as a consistency check on our computations so far, that the system of partial differential equations (4.15) is integrable. Indeed, restricted to $z_{2}=0$, the Picard-Fuchs operators satisfy the relation

$$
\mathcal{L}_{1}=\theta_{y} \mathcal{L}_{2}
$$

and clearly, the inhomogeneities are consistent with this relation. In fact, expanding

$$
\frac{1}{\sqrt{1-4 y}}=\sum_{m=0}^{\infty} \frac{\Gamma(1+2 m)}{\Gamma(1+m)^{2}} y^{m}
$$

we can integrate straightforwardly to obtain the solution at $z_{2}=0$,

$$
\mathcal{W}(y, 0)=3\left[\frac{1}{2}(\log y)^{2}+\sum_{m=1}^{\infty} \frac{\Gamma(1+2 m)}{m^{2} \Gamma(1+m)^{2}} y^{m}\right]
$$


Not surprisingly, this series can be rewritten using the dilogarithm function. But let us put off a discussion of its analytic properties as a function of $y$ until section 7.

Using (4.20) as a first step, one may find a representation of the higher order terms by solving the appropriate recursion relations. With the ansatz 1

$$
\begin{aligned}
\mathcal{W}\left(y, z_{2}\right)= & 3\left[\frac{1}{2}(\log y)^{2} \sum_{\substack{m \in 3 \mathbb{Z}, n \\
2 n \leq m \leq 3 n}} a_{m, n} y^{m} z_{2}^{n}+\log y \sum_{\substack{m \in 3 \mathbb{Z}, n \\
m \leq 3 n}} b_{m, n} y^{m} z_{2}^{n}\right. \\
& \left.+\sum_{m \in 3 \mathbb{Z}, n} c_{m, n} y^{m} z_{2}^{n}+\sum_{m \notin 3 \mathbb{Z}, n} d_{m, n} y^{m} z_{2}^{n}\right]
\end{aligned}
$$

one obtains

$$
\begin{aligned}
a_{m, n}= & \frac{\Gamma(1+2 m)}{\Gamma\left(1+\frac{m}{3}\right)^{2} \Gamma(1+m) \Gamma\left(1-\frac{m}{3}+n\right)^{2} \Gamma(1+m-2 n)} \\
b_{m, n}= & a_{m, n}\left[2 \Psi(1+2 m)-\frac{2}{3} \Psi\left(1+\frac{m}{3}\right)-\Psi(1+m)+\frac{2}{3} \Psi\left(1-\frac{m}{3}+n\right)-\Psi(1+m-2 n)\right] \\
c_{m, n}= & \frac{1}{2} a_{m, n}\left[\left(\frac{b_{m, n}}{a_{m, n}}\right)^{2}+\right. \\
& \left.4 \Psi^{\prime}(1+2 m)-\frac{2}{9} \Psi^{\prime}\left(1+\frac{m}{3}\right)-\Psi^{\prime}(1+m)-\frac{2}{9} \Psi^{\prime}\left(1-\frac{m}{3}+n\right)-\Psi^{\prime}(1+m-2 n)\right] \\
d_{m, n}= & \frac{\Gamma(1+2 m) \Gamma\left(\frac{m}{3}-n\right)^{2}}{9 \Gamma\left(1+\frac{m}{3}\right)^{2} \Gamma(1+m) \Gamma(1+m-2 n)}
\end{aligned}
$$

where $\Psi$ is the digamma function, $\Psi^{\prime}$ its derivative, and it is understood that the formulas for $b_{m, n}$ and $c_{m, n}$ require a certain limit when the arguments of the $\Gamma$-functions hit the poles. In particular, some of the restrictions on the summation indices in (4.21) are automatic. After noting that

$$
c_{0,0}=\frac{1}{2} \Psi^{\prime}(1)\left(2-\frac{4}{9}\right)=\frac{7 \pi^{2}}{54}
$$

we may rationalize the $c_{m, n}$ by subtracting the appropriate multiple of $\varpi_{0}$ from $\mathcal{W}$. It is in fact natural to rewrite this solution in a more suggestive fashion. The lift of (4.1) appropriate for our new variables $y, z_{2}$ is the table

$$
\begin{array}{c|cccccc|c}
h & -\frac{1}{3} & -\frac{1}{3} & \frac{1}{3} & \frac{1}{3} & 1 & 1 & -2 \\
l & 1 & 1 & 0 & 0 & 0 & -2 & 0
\end{array}
$$

\footnotetext{
${ }^{1}$ Summation indices will always be assumed to run over non-negative integers, with further restrictions as indicated.
} 
[This transformation is related to the following (non-integral) change of basis of cohomology

$$
\begin{aligned}
H & =3 H_{1}, \quad L=H_{1}+H_{2} \\
h=\frac{1}{3}\left(h_{1}-h_{2}\right) & =\frac{H L}{6}-\frac{2 H^{2}}{27}, \quad l=h_{2}=\frac{H^{2}}{6}-\frac{H L}{3} \\
H_{1} & =\frac{H}{3}, \quad H_{2}=L-\frac{H}{3} \\
h_{1} & \left.=3 h+l, \quad h_{2}=l\right]
\end{aligned}
$$

The generating function of solutions now takes the form

$$
\begin{aligned}
& \tilde{\varpi}\left(y, z_{2} ; H, L\right)=\sum_{m \in 3 \mathbb{Z}, n} y^{m+H} z_{2}^{n+L} \\
& \frac{\Gamma(1+2(m+H))}{\Gamma\left(1+\frac{1}{3}(m+H)\right)^{2} \Gamma(1+m+H) \Gamma\left(1-\frac{1}{3}(m+H)+n+L\right)^{2} \Gamma(1+m+H-2(n+L))}
\end{aligned}
$$

and the solution of (4.17) given above can be understood from the representation

$$
\mathcal{W}\left(y, z_{2}\right)=3\left[\frac{1}{2} \partial_{H}^{2} \tilde{\varpi}\left(y, z_{2} ; 0,0\right)+\tau\left(y, z_{2}\right)\right]
$$

where

$$
\tau\left(y, z_{2}\right)=\frac{4 \pi^{2}}{27} \sum_{m \notin 3 \mathbb{Z}, n} \frac{\Gamma(1+2 m)}{\Gamma\left(1+\frac{m}{3}\right)^{2} \Gamma(1+m) \Gamma\left(1-\frac{m}{3}+n\right)^{2} \Gamma(1+m-2 n)} y^{m} z_{2}^{n}
$$

Indeed, by construction,

$$
\begin{aligned}
& 9 \mathcal{L}_{2} \tilde{\varpi}\left(y, z_{2} ; H, L\right)=\sum_{m \in 3 \mathbb{Z}} y^{m+H} z_{2}^{L} \\
& \frac{(H+m-3 L)^{2} \Gamma(1+2(m+H))}{\Gamma\left(1+\frac{1}{3}(m+H)\right)^{2} \Gamma(1+m+H) \Gamma\left(1-\frac{1}{3}(m+H)+L\right)^{2} \Gamma(1+m+H-2 L)}
\end{aligned}
$$

When acting with $\partial_{H}^{2}$, and restricting to $H=L=0$, the terms at $m \neq 0$ would vanish because of the appearance of $\Gamma\left(1-\frac{m}{3}\right)^{2}$ in the denominator, unless both derivatives act on that factor, to yield

$$
\sum_{\substack{m \in 3 \mathbb{Z} \\ m>0}} \frac{m^{2} \Gamma(1+2 m) \Gamma\left(\frac{m}{3}\right)^{2} \cos \left(\pi \frac{m}{3}\right)^{2}}{9 \Gamma\left(1+\frac{m}{3}\right)^{2} \Gamma(1+m)^{2}} y^{m}=\sum_{\substack{m \in 3 \mathbb{Z} \\ m>0}} \frac{\Gamma(1+2 m)}{\Gamma(1+m)^{2}} y^{m}
$$


On the other hand, the term at $m=0$ gives a non-zero contribution only when both derivatives act on $(H-3 L)^{2}$. Also,

$$
\begin{aligned}
9 \mathcal{L}_{2} \tau\left(y, z_{2}\right) & =\frac{4 \pi^{2}}{27} \sum_{m \notin 3 \mathbb{Z}} \frac{m^{2} \Gamma(1+2 m)}{\Gamma\left(1+\frac{m}{3}\right)^{2} \Gamma(1+m) \Gamma\left(1-\frac{m}{3}\right)^{2} \Gamma(1+m)} y^{m} \\
& =\sum_{m \notin 3 \mathbb{Z}} \frac{\Gamma(1+2 m)}{\Gamma(1+m)^{2}} y^{m}
\end{aligned}
$$

In combination, we obtain indeed the inhomogeneity in the form (4.19). A similar computation verifies the equation for $\mathcal{L}_{1}$.

Note that we may reinstate the discrete labels on the superpotential via

$$
\mathcal{W}_{(\eta, \pm)}\left(y, z_{2}\right)= \pm \mathcal{W}_{(1,+)}\left(\eta y, z_{2}\right)
$$

\subsection{Mirror map and instanton sum}

Most of the rest of the paper is devoted to verifying that the solution of the inhomogeneous Picard-Fuchs equation has a consistent interpretation as a global holomorphic object over the entire moduli space. As an important first check, we will here show that the expansion around $y=z_{2}=0$ satisfies Ooguri-Vafa integrality, i.e., has a consistent interpretation as counting domainwall degeneracies.

The main step is to understand the mirror map. For this, note that the generating function (4.26) can of course also be used to express the solutions of the homogeneous equation. In particular, the regular and simple logarithmic solutions are

$$
\begin{aligned}
& \varpi_{0}=\tilde{\varpi}\left(y, z_{2} ; 0,0\right) \\
& \varpi_{h}=\partial_{H} \tilde{\varpi}\left(y, z_{2} ; 0,0\right)=\frac{1}{3}\left(\varpi_{h_{1}}-\varpi_{h_{2}}\right) \\
& \varpi_{l}=\partial_{L} \tilde{\varpi}\left(y, z_{2} ; 0,0\right)=\varpi_{h_{2}} .
\end{aligned}
$$

where the relations to (4.9) are dictated by (4.25). By inspecting (4.21), we may anticipate from our discussion in section 7 that the tension of a supersymmetric domainwall between vacua labelled $\eta$ and $e^{2 \pi i / 3} \eta$ behaves to leading order as $\mathcal{T}=\mathcal{W}_{\left(e^{2 \pi i / 3} \eta, \pm\right)}-\mathcal{W}_{(\eta, \pm)} \sim \varpi_{h}+\cdots$. Translated into the A-model, this means that the large volume geometry must admit a domainwall with classical tension

$$
\mathcal{T}_{\text {class }}=\frac{1}{3}\left(t_{1}-t_{2}\right) \equiv s
$$




\begin{tabular}{|l|lllllll|}
\hline$m \backslash n$ & 0 & 1 & 2 & 3 & 4 & 5 & 6 \\
\hline 0 & 0 & 1 & 0 & 0 & 0 & 0 & 0 \\
1 & 6 & 6 & 0 & 0 & 0 & 0 & 0 \\
2 & 3 & 90 & 3 & 0 & 0 & 0 & 0 \\
3 & 6 & -236 & 1012 & 6 & 0 & 0 & 0 \\
4 & 12 & -258 & 2934 & -258 & 12 & 0 & 0 \\
5 & 30 & -540 & 11016 & 11016 & -540 & 30 & 0 \\
6 & 75 & -1388 & -44274 & 348774 & 179478 & -1388 & 75 \\
\hline
\end{tabular}

Table 1: Open BPS invariant of two-parameter model.

Following [12], one may pose the problem to count the degeneracy of such domainwalls, and this information should be contained in the B-model superpotential $\mathcal{W}_{(\eta, \pm)}$. As in the previous compact examples, and in agreement with the structure found in noncompact examples [13, 40, 16], the prescription is to expand the superpotential with open string instanton corrections in terms of the classical domainwall tension corrected only by closed string instantons. In the problem at hand, we introduce

$$
\begin{aligned}
& o=\exp \left(\frac{\varpi_{h}}{\varpi_{0}}\right)=e^{2 \pi i s} \\
& q=\exp \left(\frac{\varpi_{l}}{\varpi_{0}}\right)=e^{2 \pi i t}=q_{2}
\end{aligned}
$$

The A-model expansion now takes the following form (we have omitted the constant term (4.23) )

$$
\hat{\mathcal{W}}_{(\eta, \pm)}=\frac{\mathcal{W}_{(\eta, \pm)}}{\varpi_{0}}= \pm\left[\frac{3}{2}(\log \eta o)^{2}+\sum_{\substack{(m, n) \neq(0,0) \\ k>0}} \frac{N_{m, n}}{k^{2}}(\eta o)^{k m} q^{k n}\right]
$$

The $N_{m, n}$ are indeed integer. We display the first few in table 1. Note that the invariants are symmetric under $n \rightarrow m-n$, except when $m$ is a multiple of 3 . We may improve on this after recognizing that the asymmetry is in fact a remnant of closed string instantons. More precisely, we have for $m \in 3 \mathbb{Z}$ (and $m \geq n$ )

$$
N_{m, n}-N_{m, m-n}=\frac{(2 n-m)}{2} G_{\frac{m}{3}, n-\frac{m}{3}}
$$

where $G_{i, j}$ are the standard closed string BPS invariants computed in [3]. (The $G_{i, j}$ are themselves symmetric under $j \rightarrow i-j$ and vanish outside of $0 \leq j \leq i$.) Expressed 


\begin{tabular}{|l|lllllll|}
\hline$m \backslash n$ & 0 & 1 & 2 & 3 & 4 & 5 & 6 \\
\hline 0 & 0 & 0 & 0 & 0 & 0 & 0 & 0 \\
1 & 6 & 6 & 0 & 0 & 0 & 0 & 0 \\
2 & 3 & 90 & 3 & 0 & 0 & 0 & 0 \\
3 & 6 & 388 & 388 & 6 & 0 & 0 & 0 \\
4 & 12 & -258 & 2934 & -258 & 12 & 0 & 0 \\
5 & 30 & -540 & 11016 & 11016 & -540 & 30 & 0 \\
6 & 75 & -1388 & 67602 & 348774 & 67602 & -1388 & 75 \\
\hline
\end{tabular}

Table 2: "Balanced" invariants with manifest symmetry under $m \rightarrow n-m$.

in terms of the standard variables (4.10), the combination $(2 n-m) G_{i, j}$ enters into the closed string instanton corrections of a particular 4-cycle tension

$$
2 \hat{\varpi}_{H_{2}}-\hat{\varpi}_{H_{1}}=-2 t_{1} t_{2}+\sum_{i, j, k} \frac{(2 i-j) G_{i, j}}{k^{2}} q_{1}^{k i} q_{2}^{k j}
$$

So after transforming to the new variables, the "balanced" superpotential

$$
\tilde{\mathcal{W}}=\mathcal{W}-\frac{1}{4}\left(2 \varpi_{L}-\varpi_{H}\right)=\mathcal{W}+\frac{3}{2} \partial_{H} \partial_{L} \tilde{\varpi}+\frac{1}{2} \partial_{L}^{2} \tilde{\varpi}
$$

has an A-model expansion

$$
\frac{3}{2} s^{2}+\frac{3}{2} s t+\frac{1}{2} t^{2}+\sum_{m, n, k} \frac{\tilde{N}_{m, n}}{k^{2}} o^{k m} q^{k n}
$$

with invariants $\tilde{N}_{m, n}$ that are now symmetric under $n \rightarrow m-n$, see table 2. One might try to corroborate this symmetry and the modification (4.39) in terms of monodromy calculations.

\section{Open-Closed Moduli Space of Two-parameter Model}

For the subsequent computations, it is useful to have a good global picture of the combined open-closed moduli space.

Following [3], one begins by noting that the parameter space of (4.3), spanned by $(\psi, \phi)$, is subject to a $\mathbb{Z}_{12}$ quotient generated by $(\psi, \phi) \rightarrow\left(e^{2 \pi i / 12} \psi,-\phi\right)$, since this action can be undone by the change of coordinate $x_{1} \rightarrow e^{-2 \pi i / 12} x_{1}$. The invariant combinations are

$$
\xi=\psi^{12}, \quad v=\psi^{6} \phi, \quad \zeta=\phi^{2},
$$




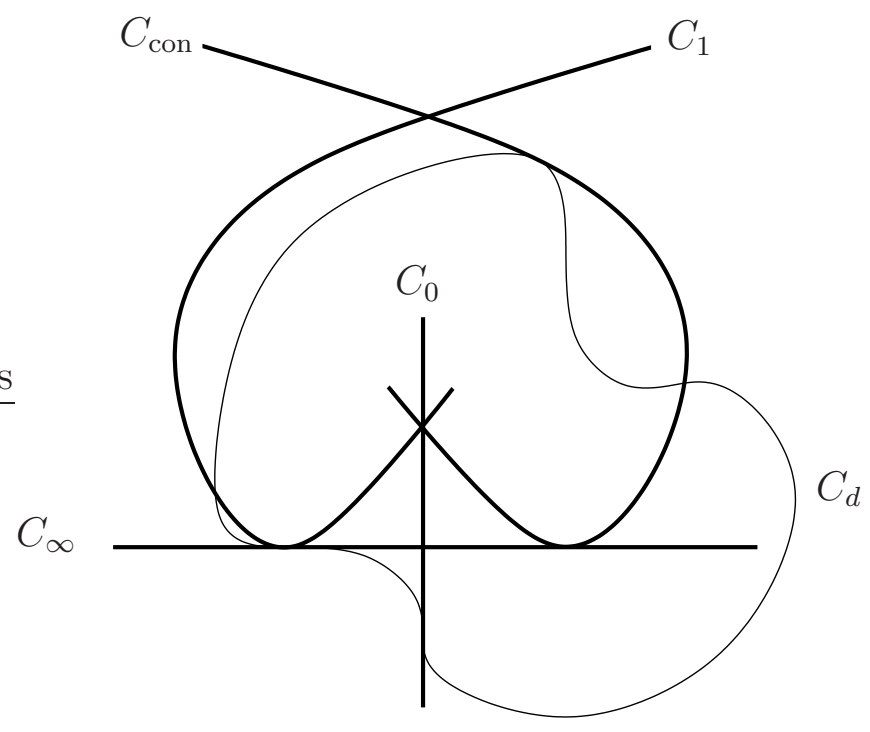

Figure 1: The compactified moduli space before blowups. The four divisors of closed string origin, depicted with a thicker line, include the large volume divisor $C_{\infty}$, the conifold locus $C_{\text {con }}$, the enhanced symmetry locus $C_{0}=\{\psi=0\}$ and the locus $C_{1}=\left\{\phi^{2}=1\right\}$. The thinner line represents the open string discriminant $C_{d}$. The orbifold point lies at the intersection of $C_{0}$ and $C_{d}$.

now subject to the relation

$$
\xi \zeta=v^{2}
$$

A first model of the compactified moduli space is obtained by viewing $(\xi, v, \zeta)$ as inhomogeneous coordinates in the patch $\tau=1$ of a copy of $\mathbb{P}^{3}$. In this compactification, there are four special loci along which the family acquires various singularities. These are

- the conifold locus $C_{\text {con }}=\{\xi+2 v+\zeta=\tau\}$, (In $\psi, \phi$ space, it is the locus $\left(\phi+\psi^{6}\right)^{2}=1$.) - the locus $C_{1}=\{\zeta=\tau\}$ (compactification of $\phi^{2}=1$ ),

- the limit of large $\psi, \phi, C_{\infty}=\{\tau=0\}$,

- the locus of enhanced symmetry $C_{0}=\{\xi=0, v=0\}(\psi=0)$.

These divisors meet at various points. Not all of these intersections are transverse, so one needs to blow up to obtain a good compactification. In practice, this is accomplished by working in the appropriate local coordinates. For example, the large complex structure point, which is (here uniquely) characterized by maximal unipotent monodromy, is hidden at the double intersection of $C_{1}$ and $C_{\infty}$. In the patch $\xi=1$, 


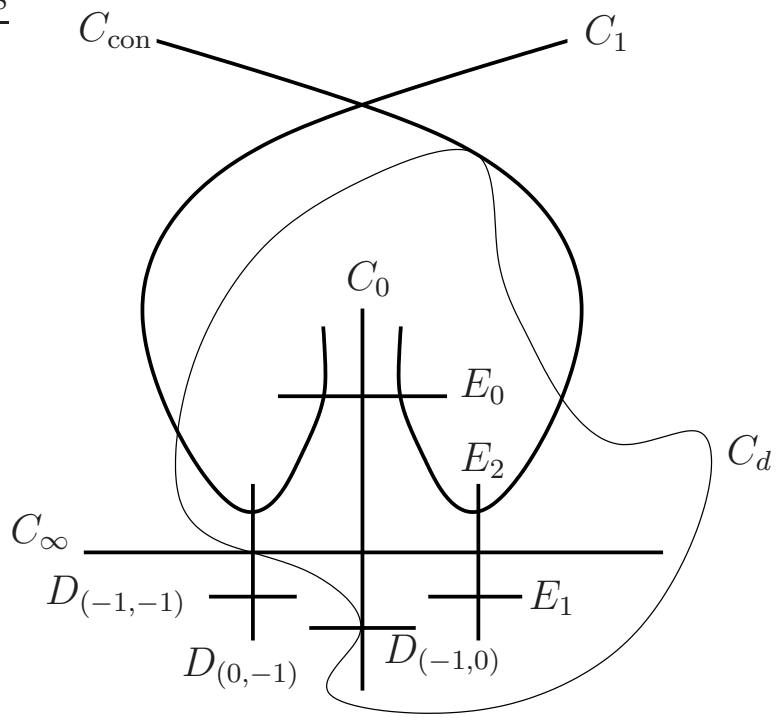

Figure 2: The moduli space blown up according to [3] such that the closed string boundary divisor has normal crossings. The large volume point lies at the intersection of $C_{\infty}=\left\{z_{1}=0\right\}$ with $D_{(0,-1)}=\left\{z_{2}=0\right\}$, and is also met by $C_{d}$.

where we can eliminate $\zeta$ using (5.2), this is the point $\tau=1 / \psi^{12}=0, v=\phi / \psi^{6}=0$. Blowing up once introduces the coordinate $\alpha=\tau / v=1 /\left(\phi \psi^{6}\right)$ on the divisor called $D_{(-1,-1)}$ in [3]. $D_{(-1,-1)}, C_{1}$ and $C_{\infty}$ now all meet at $\alpha=v=0$. A second blowup of this point inserts the divisor $D_{(-1,0)}$, with coordinate $\beta=\alpha / v=1 / \phi^{2}$ that now intersects $C_{\infty}$ transversely. The coordinates $v \sim z_{1}, \beta \sim z_{2}$ are precisely those appropriate for the closed string mirror map in the previous section.

Now let us add the D-brane. At a generic point of the moduli space, we are considering 6 disjoint curves $C_{(\eta, \pm)}$ which we think of physically as representing 6 different vacua of some $\mathcal{N}=1$ theory in 4 dimensions. The combined open-closed moduli space is thus a six-fold cover of the closed moduli. However, there are various places where some of these vacua come together, and/or are permuted in various ways under monodromy. The merging of vacua is accompanied by new light physical degrees of freedom, but is not necessary for the occurrence of monodromy. Since the monodromies should be consistent with the symmetry of the problem, there are only 3 non-trivial possibilities: $\mathrm{A} \mathbb{Z}_{2}$, a $\mathbb{Z}_{3}$, or a $\mathbb{Z}_{6} \cong \mathbb{Z}_{2} \times \mathbb{Z}_{3}$ rotation of the vacua.

It is rather straightforward to identify some of these loci from the right side of (4.15). For this, we note that $y^{3}=z_{1} / z_{2} \sim \phi^{3} / \psi^{6}$ is the coordinate on an additional blowup of the moduli space at the large complex structure point. We call this exceptional divisor 

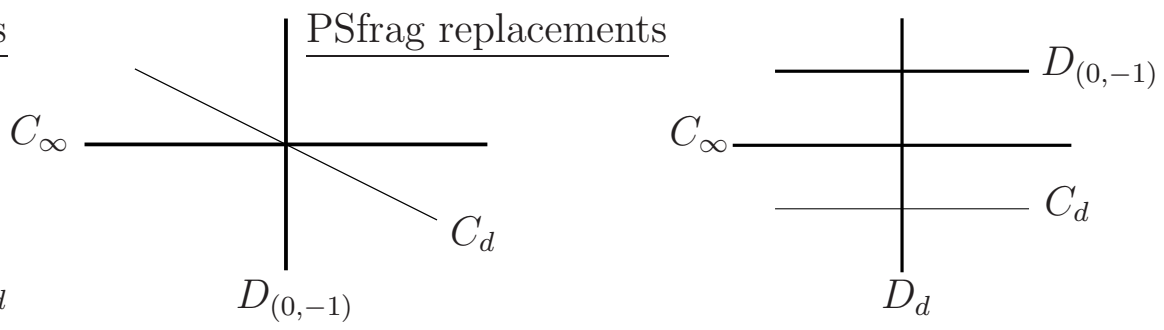

Figure 3: Close up of large volume point, with additional blowup depicted on the right. The coordinate on the exceptional divisor $D_{d}$ is denoted $y^{3}=1 / x^{3} \sim \frac{z_{1}}{z_{2}}$ in the text.

$D_{d}$. Taking a third root produces a threefold cover branched at $y=0$. We can then see

- a $\mathbb{Z}_{3}$ monodromy, acting on $(\eta, \pm) \rightarrow\left(e^{2 \pi i / 3} \eta, \pm\right)$, at $y^{3}=0$,

- a $\mathbb{Z}_{2}$ monodromy, acting as $(\eta, \pm) \rightarrow(\eta, \mp)$, at the locus $(4 y)^{3}=1$. We will denote this divisor by $C_{d}$. In the homogeneous coordinates, it is the locus

$$
C_{d}=\left\{\xi \zeta-v^{2}=0,27 v \tau+4 \zeta^{2}=0,729 \xi \tau^{2}-16 \zeta^{3}=0\right\}
$$

- a $\mathbb{Z}_{6}$ monodromy, acting as $(\eta, \pm) \rightarrow\left(e^{2 \pi i / 3} \eta, \mp\right)$ at $x \equiv y^{-1}=0$.

It is straightforward to analyze the intersections of $C_{d}$ with the loci $C_{0}, C_{1}, C_{\text {con }}$, and $C_{\infty}$ listed above. We have depicted the result in Fig. 1, and the state of affairs after the blowups of [3] in Fig. 2. We will investigate in more detail the blowup of the large complex structure point, sketched in Fig. 3, in section 7. It is likely that the other special points on $C_{d}$ might also harbor interesting physical effects.

\section{Restriction to One-parameter Model}

It is of interest to pay some more attention to the locus $\phi= \pm 1\left(z_{2}=1 / 4\right)$ of the closed string moduli space. For these values of the parameters [3], the two-parameter model $\mathbb{P}_{11226}[12]$ becomes birationally equivalent to the one-parameter model $\mathbb{P}_{111113}[2,6]$. This entails a certain relationship between the quantum geometries of the two models that provides a useful check on the calculations.

In our context, we have another reason to look at these relations. As noted in the introduction, $\mathbb{P}_{111113}[2,6]$, although its periods are hypergeometric, does not seem to possess a brane whose superpotential can be obtained by specializing $\varpi(z ; H)$ to particular simple values of $H$. (Most noticeably, $\Xi(1 / 2)=16 / \pi^{3}$.) By restricting the 
superpotential of the two-parameter model, we are able to produce a sensible extension of the Picard-Fuchs equation also in that case.

The basic relation between the two models is the identity of fundamental periods

$$
\varpi_{0}^{(1)}(z)=\varpi^{(1)}(z ; 0)=\varpi_{0}\left(2 z^{1 / 3}, 1 / 4\right)
$$

where $\varpi^{(1)}(z ; H)$ is the appropriate hypergeometric generating function. The relation for the other periods is less direct, although we still have

$$
\varpi_{1}^{(1)}(z)=3 \varpi_{h}\left(2 z^{1 / 3}, 1 / 4\right)+\frac{3}{2} \varpi_{l}\left(2 z^{1 / 3}, 1 / 4\right)
$$

It is then natural to study the restriction of the superpotential $\mathcal{W}_{(\eta, \pm)}$. Since the twice logarithmic periods of the two-parameter model do not restrict to a period of the oneparameter model, we should allow for a modification of $\mathcal{W}$ by a rational period. The best result is obtained for the combination

$$
\mathcal{W}^{(1)}(z)=\left(\mathcal{W}+\frac{1}{3} \varpi_{H}-\frac{1}{2} \varpi_{L}\right)\left(2 z^{1 / 3}, 1 / 4\right)=\left(\tilde{\mathcal{W}}+\frac{1}{12} \varpi_{H}\right)\left(2 z^{1 / 3}, 1 / 4\right)
$$

Applying the Picard-Fuchs operator of $\mathbb{P}_{111113}[2,6]$,

$$
\mathcal{L}^{(1)}=\theta^{4}-\frac{16}{3} z(6 \theta+1)(6 \theta+3)^{2}(6 \theta+5)
$$

gives the remarkable inhomogeneity

$$
\begin{aligned}
\mathcal{L}^{(1)} \mathcal{W}^{(1)}(z) & =\sum_{n=1}^{\infty} \frac{2^{n+1} \Gamma(2 n)}{27 \Gamma(n)^{2}}(10 n-9) z^{n} \\
& =\frac{4}{27} \frac{z^{1 / 3}+112 z^{2 / 3}}{\left(1-8 z^{1 / 3}\right)^{5 / 2}}
\end{aligned}
$$

One should now expect that after inserting the mirror map of the one-parameter model, $\mathcal{W}^{(1)}$ has an integer BPS expansion that moreover is related to the expansion of the two-parameter model in a simple way. Recall that in the closed string sector, one has the sum rule [3]

$$
\sum_{j=0}^{i} G_{i, j}=G_{i}^{(1)}
$$

where $G_{i}^{(1)}$ are the invariants appearing in the expansion $\left(p=\exp \left(\varpi_{1}^{(1)} / \varpi_{0}^{(1)}\right)\right)$

$$
\hat{\varpi}_{2}^{(1)}=2(\log p)^{2}+\sum_{i, k} \frac{i G_{i}^{(1)}}{k^{2}} p^{k i}
$$


For $\mathcal{W}^{(1)}$, such a relation only emerges after a modification analogous to (4.39).

$$
\tilde{\mathcal{W}}^{(1)}=\mathcal{W}^{(1)}-\frac{1}{4} \varpi_{2}^{(1)}
$$

has the A-model expansion

$$
\frac{1}{6}(\log p)^{2}+\sum_{m} \frac{N_{m}^{(1)}}{k^{2}} p^{k m / 3}
$$

These invariants now satisfy

$$
N_{m}^{(1)}=\sum_{n=0}^{m} N_{m, n}
$$

while the first few of them are

$$
N_{1}=12, N_{2}=96, N_{3}=788, N_{4}=2442, N_{5}=21012, N_{6}=481352, \ldots
$$

It will be interesting to verify the relation (6.10) by an analysis of the A-model geometry. It is also worthwhile to use the rational mapping 2 between the B-model geometries to obtain curves representative of the superpotential in the one-parameter model, and to then derive the inhomogeneity (6.5) by the Griffiths-Dwork method. This computation is, however, somewhat uncertain because the B-model geometry of the one-parameter model is singular (for generic values of the parameter) precisely at the points where the residues should be localized. If the modifications (4.39), (6.8) seem a bit ad hoc, we point out that the actual comparison of closed string invariants [3] takes place at the level of the Yukawa couplings. The open string analogue would be a comparison of Griffiths infinitesimal invariants [41].

\section{D-brane Phase Transitions at Large Volume}

In this section, we study and interpret the analytic properties of the solutions of the differential equation

$$
\theta_{y}^{2} V(y)=\frac{1}{(1-4 y)^{1 / 2}}
$$

where $\theta_{y}=y \frac{d}{d y}$. This equation is the restriction of the inhomogeneous Picard-Fuchs equation (4.17) to the locus $z_{2}=0$. The complex $y$-plane is a three-fold cover of the divisor $D_{d}$ that we introduced in section 5. There are three special points. The intersection with $C_{\infty}$ corresponds to $y=0$, the intersection with $D_{(0,-1)}$ is the point $y=\infty$, and the intersection with $C_{d}$ occurs at $y=\frac{1}{4}$.

\footnotetext{
${ }^{2}$ I am grateful to Dave Morrison and Sheldon Katz for recovering this birational equivalence from [3].
} 


\subsection{Analytic continuation via dilogarithm}

It is natural to begin and fix boundary condition at $4 y=1$. In terms of the variable

$$
w=1-4 y
$$

the differential equation (7.1) is

$$
(1-w) \partial_{w}(1-w) \partial_{w} V=w^{-1 / 2}
$$

and we pick the solution of the inhomogeneous equation that vanishes at $w=0$. This solution can be written as

$$
V(w)=\frac{1}{2}\left(\log \frac{1-\sqrt{w}}{2}\right)^{2}-\frac{1}{2}\left(\log \frac{1+\sqrt{w}}{2}\right)^{2}+\operatorname{Li}_{2} \frac{1-\sqrt{w}}{2}-\operatorname{Li}_{2} \frac{1+\sqrt{w}}{2}
$$

Here $\mathrm{Li}_{2}(z)$ is the dilogarithm. It can be defined by its power-series expansion around $z=0$

$$
\mathrm{Li}_{2}(z)=\sum_{n=1}^{\infty} \frac{z^{n}}{n^{2}}
$$

and analytically continued through the complex plane via the integral

$$
\operatorname{Li}_{2}(z)=-\int \frac{\log (1-z)}{z} d z
$$

with a branch cut from $z=1$ to $z=\infty$.

There is now little work for us to do if we are willing to refer to some standard properties of the dilogarithm. Using the transformation

$$
\mathrm{Li}_{2}(z)=-\mathrm{Li}_{2}(1-z)+\frac{\pi^{2}}{6}-\log z \log (1-z)
$$

we obtain the representation $(y=(1-w) / 4)$,

$$
V=\frac{1}{2}(\log y)^{2}-\frac{\pi^{2}}{6}-\left(\log \frac{1+\sqrt{1-4 y}}{2}\right)+2 \operatorname{Li}_{2} \frac{1-\sqrt{1-4 y}}{2}
$$

which gives the expansion around $y=0$,

$$
V=\frac{(\log y)^{2}}{2}-\frac{\pi^{2}}{6}+\sum_{m=1}^{\infty} \frac{\Gamma(1+2 m)}{\Gamma(1+m)^{2} m^{2}} y^{m}
$$

Note that this agrees with (4.20) up to a constant, which will play an important role below. On the other hand, applying the identity (valid under continuation through the upper half plane)

$$
\operatorname{Li}_{2}(1 / z)=-\operatorname{Li}_{2}(z)-\frac{\pi^{2}}{6}-\frac{1}{2}(\log (-z))^{2}
$$


we obtain (with $x=1 / y$ ),

$$
V=i \pi \ln x-\frac{1}{2}\left(\log \frac{\sqrt{x^{2}-4 x}+x}{\sqrt{x^{2}-4 x}-x}\right)^{2}-2 \operatorname{Li}_{2}\left(\frac{\sqrt{x^{2}-4 x}+x}{2}\right)
$$

which gives the expansion around $x=0$

$$
V=i \pi \log x-i \sum_{m=0}^{\infty} \frac{\Gamma(1+2 m)}{\Gamma(1+m)^{2}\left(m+\frac{1}{2}\right)^{2} 2^{4 m+1}} x^{m+1 / 2}
$$

As a consistency check, we note the transformations of $V$ under the monodromies around the three points

$$
\begin{aligned}
& y \rightarrow e^{2 \pi i} y: V \rightarrow V+2 \pi i \log y+\frac{(2 \pi i)^{2}}{2} \\
& z \rightarrow e^{2 \pi i} z: V \rightarrow-V \\
& x \rightarrow e^{2 \pi i} x: V \rightarrow-V+2 \pi i \log x+\frac{(2 \pi i)^{2}}{2}
\end{aligned}
$$

which indeed compose correctly.

\subsection{Interpretation}

The information gathered so far is sufficient to predict some non-trivial properties of the A-model geometry that is mirror to the configuration of holomorphic curves introduced in section 4. Classically, vacuum configurations of A-branes are described by Lagrangian submanifolds $L \subset X$ equipped with flat bundles. There are however, quantum corrections from worldsheet instantons wrapping holomorphic disks [10] that affect the vacuum structure in a qualitative way. Since instanton corrections die out when the Kähler moduli are taken to infinity, but are present for any finite value, the vacuum structure of A-branes can change in a discontinuous way in the large volume limit. This complication is at the heart of understanding mirror symmetry for open strings in an invariant way.

In our problem, a minimum requirement is that the A-brane should have 6 supersymmetric vacua for finite values of the Kähler moduli. We can use the structure of the superpotential, or more precisely the spectrum of domainwall tensions, to deduce what the corresponding classical configurations should look like.

Recall that in section 4 we introduced the discrete labels $(\eta, \pm)$, where $\eta$ is a third root of unity. We will presently see that because of the mixing between open strings 
and Ramond-Ramond flux degrees of freedom, it is actually more efficient to resort to $\mathbb{Z}$-valued labels. Thus we write

$$
\eta=e^{2 \pi i a / 3}, \quad \pm=e^{2 \pi i b / 2}
$$

and take $a, b \in \mathbb{Z}$. We denote the tension of supersymmetric domainwalls between adjacent vacua as

$$
\begin{aligned}
& \mathcal{T}_{2,(a, b)}=\mathcal{W}_{(a+1, b)}-\mathcal{W}_{(a, b)} \\
& \mathcal{T}_{3,(a, b)}=\mathcal{W}_{(a, b+1)}-\mathcal{W}_{(a, b)}
\end{aligned}
$$

Up to closed string periods, we can deduce the asymptotic form of $\mathcal{T}_{2}, \mathcal{T}_{3}$ from our computations in section 4. In the large volume expansion in the A-model, for $t_{1} \gg t_{2}$ we find from (4.36) (we use $s=\left(t_{1}-t_{2}\right) / 3$, and absorb all factors of $(2 \pi i)$ )

$$
\begin{aligned}
& \mathcal{T}_{2,(a, b)}=(-1)^{b} \frac{1}{3}\left(t_{1}-t_{2}+a\right)^{2}+\cdots \\
& \mathcal{T}_{3,(a, b)}=(-1)^{b} \frac{1}{3}\left(t_{1}-t_{2}+a\right)+(-1)^{b} \frac{1}{6}+\cdots
\end{aligned}
$$

We now give an interpretation of this structure from the A-model point of view, after which we will see that there are other subleading corrections to (7.16). To fix ideas somewhat more generally, we consider, in type IIA string theory compactified on a Calabi-Yau $X$, a D6-brane wrapped on a Lagrangian submanifold $L \subset X$. If we assume that $L$ is classically rigid, i.e., $b_{1}(L)=0$, possible choices of the gauge field are distinguished by the value of a discrete Wilson line $w \in H_{1}(L, \mathbb{Z}) \cong H^{2}(L, \mathbb{Z})$, or equivalently a first Chern class.

There can now be two types of domainwalls. We can change the Lagrangian submanifold to a homologous Lagrangian $L^{\prime}$, or we can change the value of the gauge field to $w^{\prime}$. The first type of transition (say around $0=x_{3} \in \mathbb{R} \subset \mathbb{R}^{3,1}$ ) is represented in space-time by a supersymmetric 4-cycle in $X \times \mathbb{R}$ that asymptotes to $L$ or $L^{\prime}$ for $x_{3} \rightarrow \pm \infty$, respectively. In the Calabi-Yau, the three-cycle sweeps out a four chain $\Gamma_{4}$, with $\partial \Gamma_{4}=L^{\prime}-L$. The classical BPS tension of such a domainwall is in the large volume limit given by

$$
\int_{\Gamma_{4}} J \wedge J
$$

where $J$ is the complexified Kähler form. To change the value of the magnetic flux $w$, we pick a relative 2-cycle $\Gamma_{2} \in H_{2}(X, L)$ that ends on $L$ in a one-cycle equivalent to 
$w^{\prime}-w$. We then wrap a D4-brane on $\Gamma_{2} \times\left\{x_{3}=0\right\}$. Its tension is classically given by

$$
\int_{\Gamma_{2}} J
$$

In our case, it is easy to distinguish the two types from (7.16). Since $J \sim t_{i}$, we see from the scaling behavior that $\mathcal{T}_{2}$ must correspond to a domainwall changing the Lagrangian submanifold, whereas $\mathcal{T}_{3}$ corresponds to a change of magnetic flux. This is consistent with having two rigid Lagrangians each with fundamental group $\mathbb{Z}_{3}$.

But there are additional constraints that we have to take into account. From the Amodel perspective, large volume monodromies, i.e., changes of $t_{i}$ by integers, must have a consistent interpretation as a symmetry of brane vacua and domainwall spectrum. As pointed out in [1], the apparent lack of periodicity of (17.16) in a can be compensated by a non-trivial action on the Ramond-Ramond fluxes. Specifically, under $a \rightarrow a+3$, $b \rightarrow b+2$, the domainwalls must return to themselves up to an integral closed string period. The possibilities here include $t_{1}, t_{2}$, and 1 , interpreted as changing the RR 4 and 6-form flux respectively. Similar reasoning implies that $\mathcal{T}_{3,(a, b)}+\mathcal{T}_{3,(a+1, b)}+\mathcal{T}_{3,(a+2, b)}$ and $\mathcal{T}_{2,(a, b)}+\mathcal{T}_{2,(a, b+1)}$ should also be integral closed string periods. (These requirements are equivalent to the superpotential having integral monodromy.)

Another constraint comes from the B-model, and the fact that the curves $C_{(\eta,+)}$ and $C_{(\eta,-)}$ merge together when $4 \eta y=1$. This means that $\mathcal{T}_{2}$ is a tensionless domainwall at the open string discriminant $C_{d}$. The calculations in the previous subsection then imply that on $D_{d}$, for $t_{1} \gg t_{2}, \mathcal{T}_{2}$ should asymptote to

$$
6 V \sim \frac{1}{3}\left(t_{1}-t_{2}\right)^{2}+\frac{1}{4}
$$

Implementing all these constraints, we find that the correct asymptotic behavior of the domainwalls is given by

$$
\begin{aligned}
& \mathcal{T}_{2,(a, b)}=(-1)^{b} \frac{1}{3}\left(t_{1}-t_{2}+a\right)^{2}+(-1)^{b} \frac{1}{4}+\cdots \\
& \mathcal{T}_{3,(a, b)}=(-1)^{b} \frac{1}{3}\left(t_{1}-t_{2}+a\right)+\frac{(-1)^{b}+1}{6}+\cdots
\end{aligned}
$$

where now the dots only contain worldsheet instanton corrections that are determined from the solution of the differential equation. For completeness, we note that the structure (7.20) can be derived from the modified superpotential

$$
\mathcal{W}_{(a, b)}=(-1)^{b} \frac{1}{6}\left(t_{1}-t_{2}+a\right)^{2}+\frac{1}{6}\left(t_{1}-t_{2}+a\right)+(-1)^{b} \frac{1}{8}+\cdots
$$


Since this differs from (4.36) only by integral periods, the modification does not interfere with the inhomogeneous Picard-Fuchs equation.

Using mirror symmetry, and the differential equations, we can analytically continue these expressions through the entire moduli space. This could be used to check integrality of the monodromies around the small volume phases of the closed string geometry.

For the moment, we will however restrict attention to the behavior under analytic continuation on the exceptional large volume divisor $D_{d}$. Notice that as we move towards $C_{d}, \mathcal{T}_{2}$ decreases. This means that under this deformation of symplectic structure of $X$, the two Lagrangians classically approach each other (this is another indication that the Lagrangians should not be real slices). Simultaneously however, worldsheet instanton corrections become strong, spoiling the geometric picture. Another semiclassical regime emerges for $t_{2} \gg t_{1}$, i.e., close to $D_{(0,-1)}$. What can we say about the Lagrangian geometry in this phase? From (7.12), we find the asymptotic behavior in this regime

$$
\begin{aligned}
& \tilde{\mathcal{T}}_{2,(a, b)}=(-1)^{b}\left(t_{2}-t_{1}\right)+\cdots \\
& \tilde{\mathcal{T}}_{3,(a, b)}=(-1)^{b} \frac{1}{2}+\frac{1}{6}+\cdots
\end{aligned}
$$

where the dots again only contain non-perturbative corrections. We see from these expressions that there should be only a single Lagrangian submanifold $\tilde{L}$ relevant in this phase. The domainwall $\mathcal{T}_{2}$ must correspond to a holomorphic disk ending on $\tilde{L}$. Moreover, the domainwall $\mathcal{T}_{3}$ classically has vanishing tension (in general, the constant non-integral terms in the above superpotentials are expected to arise from perturbative worldsheet corrections). Thus it appears that in this regime, although all closed string worldsheet instanton corrections are small, the vacuum structure on $\tilde{L}$ cannot be understood classically.

There is a hopefully more invariant way to characterize the occurrence of this phase transition. The asymptotic form of $\mathcal{T}_{3}$ for $t_{1} \gg t_{2}$ implies that the Lagrangians $L$, $L^{\prime}$ admit the boundary of holomorphic disks whose symplectic area scales as $\left(t_{1}-\right.$ $\left.t_{2}\right) / 3$. (This is precisely the variable in which we have expanded the superpotential in section 4. Note that the notion is well-defined since when $H_{1}(L)$ is torsion we can invert the map $H_{2}(X) \rightarrow H_{2}(X, L)$ over the rationals.) Such holomorphic disks then introduce additional walls in the Kähler cone, across which the disks undergo a transition reminiscent of a "flop". (The Lagrangian in the other phase does not seem 
to have domainwalls scaling as $\left(t_{2}-t_{1}\right) / 3$. The instanton corrections suggest that the disk is nevertheless still present.) Note that flop transitions of holomorphic disks under variation of open string moduli have been observed in [13, 40, 42, 43]. A transition between 4-chain and 2-chain domainwalls under variation of only closed string moduli was described in [20], but involved continuation through small volume phases.

\section{Outlook}

In this work, we have computed D-brane contributions to the spacetime superpotential for bulk fields in several compact Calabi-Yau geometries. Our B-model results are holomorphic invariants of the underlying quantum D-brane geometry and we have interpreted the results in appropriate semi-classical regimes in the A-model. Among our findings is an interesting phase transition under which the classical topology of the Lagrangian geometry and associated domainwalls changes. Among possible future directions, let us mention the following three.

First of all, it will be interesting to see whether the vacuum structure in the regime $t_{2} \gg t_{1}$ can be understood in semi-classical terms in the A-model. This is likely to involve dynamical open string moduli, which we have suppressed in the entire discussion.

Secondly, it would be interesting to repeat the analysis for other multi-parameter models, to see how much of the structure survives. Residue computations on the twoparameter models $\mathbb{P}_{11222}[8]$ and $\mathbb{P}_{11669}[18]$ give results very similar to (4.14). However, the quadratic equation (4.13) is replaced by a quartic and cubic equation, respectively. This makes the solution of the extended Picard-Fuchs equations slightly more complicated.

Thirdly, one could investigate the structure of loop amplitudes in these models, using the extended holomorphic anomaly equations of [41, 38]. This is interesting because the D-branes that we have studied here presumably do not arise as fixed point sets of anti-holomorphic involutions in the A-model. As a result, they should offer a greater degree of flexibility in implementing the topological tadpole cancellation condition of [38].

Acknowledgments I would like to thank Manfred Herbst, Wolfgang Lerche, David Morrison, and Edward Witten for valuable discussions and comments. 


\section{References}

[1] J. Walcher, "Opening Mirror Symmetry on the Quintic," Comm. Math. Phys. 276 671689 (2007) arXiv:hep-th/0605162

[2] D. R. Morrison and J. Walcher, "D-branes and Normal Functions," Adv. Theor. Math. Phys. 13, 553 (2009) arXiv:0709.4028 [hep-th]].

[3] P. Candelas, X. De La Ossa, A. Font, S. H. Katz and D. R. Morrison, "Mirror symmetry for two parameter models. I," Nucl. Phys. B 416, 481 (1994) arXiv:hep-th/9308083.

[4] P. Candelas, A. Font, S. H. Katz and D. R. Morrison, "Mirror symmetry for two parameter models. 2," Nucl. Phys. B 429, 626 (1994) arXiv:hep-th/9403187.

[5] S. Hosono, A. Klemm, S. Theisen and S. T. Yau, "Mirror Symmetry, Mirror Map And Applications To Calabi-Yau Hypersurfaces," Commun. Math. Phys. 167, 301 (1995) arXiv:hep-th/9308122.

[6] S. Hosono, A. Klemm, S. Theisen and S. T. Yau, "Mirror symmetry, mirror map and applications to complete intersection Calabi-Yau spaces," Nucl. Phys. B 433, 501 (1995) arXiv:hep-th/9406055.

[7] E. Witten, "Chern-Simons Gauge Theory As A String Theory," Prog. Math. 133, 637 (1995) arXiv:hep-th/9207094.

[8] C. Vafa, "Extending mirror conjecture to Calabi-Yau with bundles," arXiv:hep-th/9804131.

[9] I. Brunner, M. R. Douglas, A. E. Lawrence and C. Romelsberger, "D-branes on the quintic," JHEP 0008, 015 (2000) arXiv:hep-th/9906200.

[10] S. Kachru, S. H. Katz, A. E. Lawrence and J. McGreevy, "Open string instantons and superpotentials," Phys. Rev. D 62, 026001 (2000) arXiv:hep-th/9912151.

[11] C. I. Lazaroiu, "String field theory and brane superpotentials," JHEP 0110, 018 (2001) arXiv:hep-th/0107162.

[12] H. Ooguri and C. Vafa, "Knot invariants and topological strings," Nucl. Phys. B 577, 419 (2000) arXiv:hep-th/9912123.

[13] M. Aganagic and C. Vafa, "Mirror symmetry, D-branes and counting holomorphic discs," arXiv:hep-th/0012041.

[14] M. Aganagic, A. Klemm, M. Marino and C. Vafa, "The topological vertex," Commun. Math. Phys. 254, 425 (2005) arXiv:hep-th/0305132.

[15] P. Mayr, "N = 1 mirror symmetry and open/closed string duality," Adv. Theor. Math. Phys. 5, 213 (2002) arXiv:hep-th/0108229.

[16] W. Lerche, P. Mayr and N. Warner, "Holomorphic N = 1 special geometry of openclosed type II strings," arXiv:hep-th/0207259; "N = 1 special geometry, mixed Hodge variations and toric geometry," arXiv:hep-th/0208039. 
[17] K. Fukaya, Y.-G. Oh, H. Ohta and K. Ono, "Lagrangian intersection Floer theoryanomaly and obstruction", preprint (2000)

[18] P. S. Aspinwall and S. H. Katz, "Computation of superpotentials for D-Branes," Commun. Math. Phys. 264, 227 (2006) arXiv:hep-th/0412209].

[19] R. Pandharipande, J. Solomon and J. Walcher, "Disk enumeration on the Quintic 3fold," J. Amer. Math. Soc. 21, 1169-1209 (2008) arXiv:math/0610901

[20] D. Krefl and J. Walcher, "Real Mirror Symmetry for One-parameter Hypersurfaces," JHEP 0809, 031 (2008) arXiv:0805.0792 [hep-th]].

[21] J. Knapp and E. Scheidegger, "Towards Open String Mirror Symmetry for OneParameter Calabi-Yau Hypersurfaces," arXiv:0805.1013 [hep-th],

[22] H. Jockers and M. Soroush, "Effective superpotentials for compact D5-brane Calabi-Yau geometries," arXiv:0808.0761 [hep-th].

[23] T. W. Grimm, T. W. Ha, A. Klemm and D. Klevers, "The D5-brane effective action and superpotential in N=1 compactifications," arXiv:0811.2996 [hep-th],

[24] M. Alim, M. Hecht, P. Mayr and A. Mertens, "Mirror Symmetry for Toric Branes on Compact Hypersurfaces," arXiv:0901.2937 [hep-th].

[25] H. Jockers and M. Sorous, "Relative periods and open-string integer invariants for a compact Calabi-Yau hypersurface," arXiv:0904.4674 [hep-th]

[26] A. Schwarz and V. Vologodsky, "Integrality theorems in the theory of topological strings," arXiv:0807.1714 [hep-th],

[27] M. Kerr, G. Pearlstein, "An Exponential History of Functions with Logarithmic Growth," arXiv:0903.4903 [math.AG]

[28] P. S. Aspinwall, B. R. Greene and D. R. Morrison, "Calabi-Yau moduli space, mirror manifolds and spacetime topology change in string theory," Nucl. Phys. B 416, 414 (1994) arXiv:hep-th/9309097.

[29] E. Witten, "Phases of $\mathrm{N}=2$ theories in two dimensions," Nucl. Phys. B 403, 159 (1993) arXiv:hep-th/9301042.

[30] B. R. Greene and M. R. Plesser, "Duality in Calabi-Yau moduli space," Nucl. Phys. B 338, 15 (1990).

[31] V. Batyrev, "Dual Polyhedra and Mirror Symmetry for Calabi-Yau Hypersurfaces in Toric Varieties," J. Alg. Geom. 3, 493-535 (1994) arXiv:alg-geom/9310003

[32] P. A. Griffiths, "On the periods of certain rational integrals. I, II," Ann. of Math. (2) 90 460-495 (1969); ibid. (2) 90 496-541 (1969)

[33] P. Berglund, P. Candelas, X. De La Ossa, A. Font, T. Hubsch, D. Jancic and F. Quevedo, "Periods for Calabi-Yau and Landau-Ginzburg vacua," Nucl. Phys. B 419, 352 (1994) arXiv:hep-th/9308005. 
[34] V. V. Batyrev and D. van Straten, "Generalized hypergeometric functions and rational curves on Calabi-Yau complete intersections in toric varieties," Commun. Math. Phys. 168, 493 (1995) arXiv:alg-geom/9307010.

[35] J. Knapp and E. Scheidegger, "Matrix Factorizations, Massey Products and F-Terms for Two-Parameter Calabi-Yau Hypersurfaces," arXiv:0812.2429 [hep-th].

[36] M. Herbst, K. Hori and D. Page, "Phases Of N=2 Theories In 1+1 Dimensions With Boundary," arXiv:0803.2045 [hep-th].

[37] A. Libgober, J. Teitelbaum, "Lines on Calabi-Yau complete intersections, mirror symmetry, and Picard-Fuchs equations," Int. Math. Res. Not. 1993, 29-39 (1993)

[38] J. Walcher, "Evidence for Tadpole Cancellation in the Topological String," Comm. Num. Th. Phys. 3, 111-172 (2009) arXiv:0712.2775 [hep-th]].

[39] A. Klemm and S. Theisen, "Mirror maps and instanton sums for complete intersections in weighted projective space," Mod. Phys. Lett. A 9, 1807 (1994) arXiv:hep-th/9304034].

[40] M. Aganagic, A. Klemm and C. Vafa, "Disk instantons, mirror symmetry and the duality web," Z. Naturforsch. A 57, 1 (2002) arXiv:hep-th/0105045.

[41] J. Walcher, "Extended Holomorphic Anomaly and Loop Amplitudes in Open Topological String," arXiv:0705.4098 [hep-th],

[42] W. Lerche and P. Mayr, "On N = 1 mirror symmetry for open type II strings," arXiv:hep-th/0111113.

[43] M. Herbst, W. Lerche and D. Nemeschansky, "Instanton geometry and quantum A(infinity) structure on the elliptic curve," arXiv:hep-th/0603085. 